\title{
Optimal Tracking Control Design of Quantum Systems via Tensor Formal Power Series Method
}

\author{
Bor-Sen Chen ${ }^{1, *}$, Wen-Hao Chen ${ }^{1,2},{\text { Fan } \mathrm{Hsu}^{1} \text { and Weihai Zhang }}^{3}$ \\ ${ }^{I}$ Department of Electrical Engineering, National Tsing-Hua University, Hsinchu, 30013, Taiwan. ${ }^{2}$ Department of Elec- \\ trical Engineering, Hsiuping Institute of Technology, Taichung, 41280, Taiwan. ${ }^{3}$ Information and Control Research Cen- \\ ter Shenzhen Graduate School, Harbin Institute of Technology, HIT Campus, Shenzhen University Town, Xili, Shenzhen \\ 518055, P.R. China
}

\begin{abstract}
In this study, in order to generate a sequence of desired quantum states (or quantum bits) for quantum communication and computation, it is more appealing to formulate a quantum control system as a bilinear state reference tracking system. An optimal tracking control is proposed to achieve the state-tracking by solving a Hamilton-Jacobi equation (HJE). In order to avoid the difficulty in solving the HJE with a closed-form solution, the technique of formal tensor power series is employed to treat with the HJE to obtain the optimal tracking control in quantum systems from the approximate design perspective. If the quantum system suffers from stochastic parameter variations, it could be modeled as state-dependent noise. In the situation, stochastic optimal tracking control design is also developed for quantum systems. Finally, several examples are given to illustrate the design procedure and to confirm the performance of the proposed tracking control method.
\end{abstract}

\section{INTRODUCTION}

Modeling and control of quantum mechanical systems have been discussed since 1980s [1-3]. One needs to control the dynamics of reacting atoms and molecules at the microscopic level, which needs the knowledge of quantum mechanics for exact understanding and description of the dynamics. Optimal control has been used for quantum mechanical system [4-7] by solving the two-point boundary problem via Lagrange multiplier method. In the early 1980s, chemists have tried to control chemical reactions by properly arranging electromagnetic field $[5,6,8]$ to increase the probability of a favorable chemical reaction. A feed-forward control was developed based on the method of "inverse problem" via the selection of Hamiltonian [9]. The chemical experiments on the interaction between the electromagnetic field and two or three level atomic systems led to one possible generalization of controllability of quantum mechanical systems [10-13]. Since a quantum mechanical system can be regarded as a family of unitary operators, the controllability is based on the unitary representation of Lie group. This technique has resolved the quantum feed-forward control into a unitary operator construction problem.

In order to treat the fluctuations of photocurrent in a quantum mechanical system, feedback control for quantum systems appeared in quantum optics [14-16]. Feedback control via the transfer function method was developed for quantum mechanical $[17,18]$. The stochastic Schrodinger equation was introduced so that one can control quantum systems under noise [19-23].

\footnotetext{
*Address correspondence to this author at the Department of Electrical Engineering, National Tsing-Hua University, Hsinchu, 30013, Taiwan; Tel: +8863573 1155; Fax: +88635715971;

E-mail: bschen@ee.nthu.edu.tw
}

Recently, progress in quantum electronics has revealed the possibility of quantum information technologies, which are expected to eliminate the bottleneck of modern communications and computation $[17,18]$. In the conventional optimal control of quantum systems $[4,5,7]$ one needs to solve two-point boundary equations to achieve the optimal control to steer the quantum state to approach an equilibrium point. It is not easy for a quantum system to track a sequence of desired states. In order to make quantum systems useful in communication and computation, it is assumed that we can specify a sequence of desired quantum states whenever we need it, no matter how the environment of the quantum system would be. In other words, it is presumed that the quantum state can be controlled to generate a sequence of desired quantum bits for the use of communications and computation [17], i.e., the two-level spin quantum system should track instantly a sequence of desired states (or quantum bits) which are needed for communications and computation. This presumption is far from trivial by taking into account the fact that the quantum systems sometimes entangle with the environmental systems, which result in a noisy information resource. In the situation, robust tracking control is necessary to guarantee the quantum system to track any desired quantum state we need in the presence of uncertainties or noises. Therefore, the proposed stochastic optimal tracking control is different from the conventional optimal controls for quantum systems. In short, the goal of our proposed approach is to achieve the optimal tracking control of quantum systems whether uncertainties or noises are presented or not so that it can also generate a sequence of desired quantum bits for the use of communications and computation.

In this study, the quantum system is modeled by a bilinear state space model. The desired quantum state to be tracked is from a reference model. Then a controller is specified to make the quantum system track optimally a desired 
reference state. In the conventional optimal control in quantum system, one needs to solve a two-point boundary problem by the Lagrange multiplier method [4-7]. In the proposed optimal tracking control of quantum system, Bellman dynamic programming equation is used in this study. Based on Bellman dynamic programming [24], the proposed optimal tracking control problem of quantum mechanical systems needs to solve a HJE. Because the HJE is a nonlinear partial differential equation, it can not be easily solved analytically to obtain a closed-form optimal tracking control design of quantum mechanical systems. For the convenience of design, a tensor formal power series approach is employed to solve the HJE approximately. After solving the tensor formal power series for the HJE [25], we can approximate the optimal tracking control of quantum mechanical systems. If the parameter variation noise and approximation error can be modeled by a stochastic process, then the controlled quantum mechanical system can be considered as a stochastic state reference tracking system. In the situation, an optimal stochastic tracking control is also developed to achieve a desired reference state tracking. Because the parameter variation noise and modeling error always exist in practical case, the proposed stochastic tracking control design for quantum mechanical system is much potential for practical application.

Because the quantum state will collapse to some eigenstate when we observe it, we find the optimal control input by simulating the feedback system model with computer within which there is a model to represent the quantum mechanical system, and enforce actually the control with open loop on the actual quantum mechanical system $[4,5]$. The systematic block diagram is shown in Fig. (1). Tracking control signal is generated by the controller using the information of tracking error to make the state $x(t)$ of a quantum system track a desired state $x_{d}(t)$ optimally.

In summary, the main contributions of this paper are as follows. First, our control approach can track any desired reference state, whereas the conventional optimal quantum control in $[4-7,26-28]$ only can regulate the quantum system to a fixed quantum system state but can not track any desired reference state. Second, a tensor formal power series approach is employed to solve the HJE approximately, since it can not be easy to solve a HJE analytically. Third, our control approach can be suitable for an optimal stochastic tracking control while the parameter variation noise and modeling error exist.

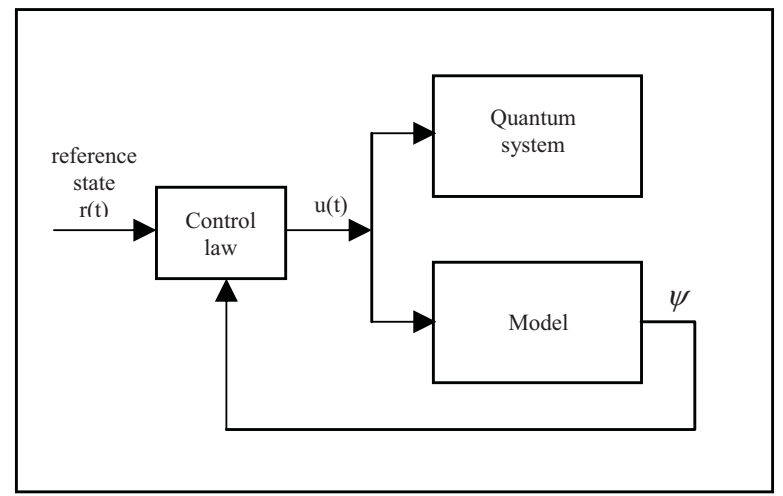

Fig. (1). Systemic block diagram of tracking control of quantum systems.
The topics in later sections are summarized as follows. The controlled quantum system is described in the state space in section II. The optimal control tracking control strategy is proposed in section III, whereas the optimal stochastic tracking control is further discussed in section IV. The techniques of tensor formal power series will be employed to solve the nonlinear partial differential HJE and then the optimal tracking control is expressed with a tensor formal power series in section V. In section VI, several simulation examples are given to illustrate the design procedure and to confirm the tracking performance of the proposed control design method. Finally, a conclusion is made in section VII.

\section{STATE SPACE DESCRIPTION OF QUANTUM MECHANICAL SYSTEMS}

Consider a quantum mechanical system to be controlled as follows [3-5]:

$$
i \hbar|\dot{\Phi}(t)\rangle=\hat{H}_{0}|\Phi(t)\rangle
$$

where $|\Phi(t)\rangle$ is the state of the quantum system, defined in a (finite or infinite-dimensional) complex Hilbert space $\mathcal{H} . \hbar$ is the Planck's constant divided by $2 \pi . \hat{H}_{0}$ is a Hamiltonian operator representing the energy of the system. The energy includes the kinetic energy and potential energy. By convention, we normalize $\langle\Phi(t) \mid \Phi(t)\rangle=1, \quad \forall t \geq 0 \quad$ (Merzbacher 1998) [28]. The result conforms to the probability concept, i.e., $|\Phi(t)\rangle$ displays the distribution of the eigenstates of the Hamiltonian operator. The eigenstates satisfy [29],

$$
\hat{H}_{0}\left|\varphi_{i}\right\rangle=E_{i}\left|\varphi_{i}\right\rangle, \quad i=1,2,3, \ldots .
$$

and are orthogonal to each other, where $\left|\varphi_{i}\right\rangle$ is the $i$-th eigenstate of the quantum system, and $E_{i}$ means the eigenenergy of the $i$-th eigenstate $\left|\varphi_{i}\right\rangle$. Consider a quantum system with normalized eigenstates $\left|\varphi_{1}\right\rangle,\left|\varphi_{2}\right\rangle,\left|\varphi_{3}\right\rangle$, with which the state $|\Phi(t)\rangle$ can be decomposed as [29]:

$|\Phi(t)\rangle=c_{1}(t)\left|\varphi_{1}\right\rangle+c_{2}(t)\left|\varphi_{2}\right\rangle+c_{3}(t)\left|\varphi_{3}\right\rangle+\ldots .$.

where $c_{i}(t)$ is the probability amplitude of the $i$-th eigenstate $\left|\varphi_{i}\right\rangle$ and $\left|c_{i}(t)\right|^{2}$ expresses its probability. Because $\langle\Phi(t) \mid \Phi(t)\rangle=1$, we can find that $\sum\left|c_{i}(t)\right|^{2}=1$.

The control of a quantum system is generated by the external potential fields, and the fields can influence the Hamiltonian operator. The controlled quantum system is described as follows [26, 30-34]

$$
i \hbar|\dot{\Phi}(t)\rangle=\left(\hat{H}_{0}+\sum_{k=1}^{m} u_{k} \hat{H}_{k}\right)|\Phi(t)\rangle
$$

where $\hat{H}_{k}$ are the external Hamiltonian operators that are influenced by the external electromagnetic field, and $u_{k}(t)$ are related to the strength of the electromagnetic field. We 
can control the quantum state to any desired state by designing $u_{k}(t)$ as control inputs.

In order to apply the control law in the quantum system, we need first to remodel the Schrödinger equation in the matrix representation [29] to let the state and eigenstates of the quantum system be the form of vectors, and then the Hamiltonian operator be the form of matrices. The remodeled dynamic system in the matrix representation is

$$
i \hbar \dot{\psi}(t)=\left(H_{0}+\sum_{k=1}^{m} u_{k}(t) H_{k}\right) \psi(t)
$$

where the state vector $\psi(t)=\left[\begin{array}{lll}c_{1}(t) & c_{2}(t) & \cdots\end{array}\right]^{T}$, and $H_{0}, H_{k}, k=1, \ldots, m$ are Hermitian matrices.

If the quantum system to be controlled is infinitedimensional or high-dimensional, for the convenience of control design, we only consider to control a small number of eigenstates whose eigenvalues are close to each other and far away from those of the other rest eigenstates. In the situation, we use a weaker control to achieve a near desired state, not necessarily to arrive at the far eigenstates with larger eigenvalues, which are truncated in the design problem. Therefore we can just discuss the system state that changes on the space which is composed of partial eigenstates, and the dimension of the quantum system in (4) is reduced significantly.

Generally, the control objective in quantum systems is to control the probabilities $\left|c_{i}(t)\right|^{2}, \quad \mathrm{i}=1,2, \ldots$ for which the quantum system is respectively at the eigenstates $\left|\varphi_{i}\right\rangle, i=1,2, \ldots$. We will develop a tracking control law by using the optimal control method. Obviously, the desired state control design in quantum systems can be formulated as an optimal tracking problem.

\section{OPTIMAL TRACKING CONTROL IN QUANTUM SYSTEMS}

Consider a quantum system of dimension $n$. The dynamic model of the quantum system is of the following form

$$
i \hbar \dot{\psi}(t)=\left(H_{0}+\sum_{k=1}^{m} u_{k}(t) H_{k}\right) \psi(t)
$$

where $\psi(t) \in C^{n} ; H_{0} \in C^{n \times n} ; H_{k} \in C^{n \times n} ; u_{k} \in R, k=1,2, \cdots, m$, and $H_{0}, H_{k}$ are Hermitian matrices. This quantum system state is defined in complex space. In order to decompose the quantum mechanical equation into real and imaginary parts, the following real system state vector and matrices are employed to denote both real part and imaginary part of quantum system in (5):

$$
\begin{aligned}
& x(t)=\left[\begin{array}{l}
\operatorname{Re}[\psi(t)] \\
\operatorname{Im}[\psi(t)]
\end{array}\right], G_{0}=\frac{1}{\hbar}\left[\begin{array}{cc}
\operatorname{Im}\left[H_{0}\right] & \operatorname{Re}\left[H_{0}\right] \\
-\operatorname{Re}\left[H_{0}\right] & \operatorname{Im}\left[H_{0}\right]
\end{array}\right], \\
& G_{k}=\frac{1}{\hbar}\left[\begin{array}{cc}
\operatorname{Im}\left[H_{k}\right] & \operatorname{Re}\left[H_{k}\right] \\
-\operatorname{Re}\left[H_{k}\right] & \operatorname{Im}\left[H_{k}\right]
\end{array}\right], k=1,2, \ldots, m
\end{aligned}
$$

In this situation, the complex quantum mechanical dynamic equation (5) can be equivalently represented by the following real quantum mechanical system:

$$
\dot{x}=G_{0} x+\sum_{k=1}^{m} u_{k} G_{k} x
$$

where $\quad x \in R^{2 n} ; G_{0} \in R^{2 n \times 2 n} ; G_{k} \in R^{2 n \times 2 n}, u_{k} \in R, k=1,2$, $\cdots, m$. Since $H_{0}, H_{k}$ are Hermitian, $G_{0}, G_{k}$ are skewsymmetric matrices with all their eigenvalues in the imaginary axis. Furthermore, it fits in with $\|x\|^{2}=1$, where $\|x\|^{2}=x_{1}^{2}+x_{2}^{2}+\ldots .+x_{2 n}^{2}$.

Assume the desired state to be steered is $x_{d} \in R^{2 n}$ with $\|x\|_{d}^{2}=1$. Before the optimal tracking control is developed for the quantum system (7) to achieve the desired state, the tracking cost function is defined as follows:

$$
\begin{aligned}
& J=\left(x\left(t_{f}\right)-r\left(t_{f}\right)\right)^{T} F_{o}\left(x\left(t_{f}\right)-r\left(t_{f}\right)\right)+ \\
& \int_{0}^{t_{f}}\left((x-r)^{T} Q_{o}(x-r)+\sum_{k=1}^{m} \rho_{k} u_{k}^{2}\right) d t
\end{aligned}
$$

where $F_{0}$ and $Q_{0}$ are symmetric positive definite weighting matrices, and $\rho_{k}$ are positive numbers.

The reference model is defined as

$$
\dot{r}=0, r(0)=x_{d}
$$

i.e., the reference state could be any desired quantum state $x_{d}$, or $r(t) \equiv x_{d}$ for $t \geq 0$.

The optimal reference tracking control problem is to specify $u_{1}, u_{2}, \ldots, u_{m}$ such that the cost function $J$ in (8) is minimized. In the conventional optimal quantum control in [26], only the total control energy $\int_{0}^{t_{f}} \sum_{k=1}^{m} \rho_{k} u_{k}^{2} d t$ is minimized so that it is not suitable for tracking design of quantum systems. However, in this study, the control strategy is to minimize the terminal tracking error, the tracking path error and the control effort simultaneously. The tradeoffs are dependent on the specification of weightings $F_{0}, Q_{0}$ and $\rho_{k}$ by designers. This optimal tracking control is suitable not only for the two-level quantum system but also for other multi-level quantum systems. It is difficult to find the optimal solution of a nonlinear tracking control system directly. It could be transformed to the following optimal state regulation problem.

Let $\eta=\left[\begin{array}{l}x \\ r\end{array}\right] \in R^{4 n}, \tilde{x}:=x-r=\left[\begin{array}{ll}I_{2 n} & -I_{2 n}\end{array}\right] \eta$

where $\eta$ is the augmented state and $\tilde{x}$ is the tracking error.

Then the optimal tracking system (7) and (8) can be augmented as the following optimal state regulation problem: 
$\dot{\eta}=A \eta+\sum_{k=1}^{m} u_{k} B_{k} \eta \quad k=1,2, \ldots . ., m$

$J=\eta_{f}^{T} F \eta_{f}+\int_{0}^{t_{f}}\left(\eta^{T} Q \eta+\sum_{k=1}^{m} \rho_{k} u_{k}^{2}\right) d t$

here $A=\left[\begin{array}{cc}G_{0} & 0 \\ 0 & 0\end{array}\right], B_{k}=\left[\begin{array}{cc}G_{k} & 0 \\ 0 & 0\end{array}\right], \quad k=1,2, \ldots ., m$

$F=\left[\begin{array}{c}I_{2 n} \\ -I_{2 n}\end{array}\right] F_{0}\left[\begin{array}{ll}I_{2 n} & -I_{2 n}\end{array}\right], Q=\left[\begin{array}{c}I_{2 n} \\ -I_{2 n}\end{array}\right] Q_{0}\left[\begin{array}{ll}I_{2 n} & -I_{2 n}\end{array}\right]$.

The optimal regulation problem of quantum mechanical system in (10) and (11) is to specify control variables $u_{k}(t), k=1,2, \ldots, m$ to make $J$ as small as possible. This is a bilinear optimal regulation problem, the Bellman dynamic programming techniques [24] in nonlinear optimal regulation problem will be employed to treat this optimal control problem of quantum systems. Then we can have the following result.

Theorem 1: The optimal tracking control of the quantum mechanical system (7) is given as

$u_{k}^{o}=-\frac{1}{2 \rho_{k}} \frac{\partial V^{T}}{\partial \eta} B_{k} \eta, k=1,2, \ldots ., m$

where $V(\eta, t)>0$ is solved from the following nonlinear partial differential $\mathrm{HJE}$

$-\frac{\partial V}{\partial t}=\eta^{T} Q \eta+\frac{\partial V^{T}}{\partial \eta} A \eta-\sum_{k=1}^{m} \frac{1}{4 \rho_{k}} \frac{\partial V^{T}}{\partial \eta} B_{k} \eta \eta^{T} B_{k}^{T} \frac{\partial V}{\partial \eta}$

$V\left(\eta_{f}, t_{f}\right)=\eta_{f}^{T} F \eta_{f}$

Proof: See Appendix 1.

\section{STOCHASTIC TRACKING CONTROL UNDER STOCHASTIC PARAMETER PERTURBATION}

In the controlled mechanical system (4), the nominal Hamiltonian operator $H_{0}$ is defined by the kinetic energy and potential energy in the invariant potential field. Sometimes, there is systemic variation or uncertainty, which is due to temperature variation, modeling error, change of magnetic moment of nucleus, perturbation of equipment, etc.. The uncertain variation $\Delta H$ could be decomposed as $H_{n} n(t)$, where the stochastic part is absorbed by the zero mean white noise $n(t)$ with unit variance and the deterministic part is absorbed by $H_{n}$. In the situation, the dynamic quantum system (4) could be modified as the following Itô differential system

$i \hbar d \psi(t)=\left(H_{0}+\sum_{k=1}^{m} u_{k}(t) H_{k}\right) \psi(t) d t+H_{n} \psi(t) d W$

where $d W=n(t) d t$ and $W$ is the standard Brownian motion process, and $H_{n} d W$ means the stochastic perturbation Hamiltonian operator. By the similar transformation procedure, the quantum mechanical system with stochastic parameter perturbation could be modified from (10) as follows

$$
d \eta=\left(A \eta+\sum_{k=1}^{m} u_{k}(t) B_{k} \eta\right) d t+C \eta d W
$$

where $C=\left[\begin{array}{cc}G_{n} & 0 \\ 0 & 0\end{array}\right]$ with

$$
G_{n}=\frac{1}{\hbar}\left[\begin{array}{cc}
\operatorname{Im}\left[H_{n}\right] & \operatorname{Re}\left[H_{n}\right] \\
-\operatorname{Re}\left[H_{n}\right] & \operatorname{Im}\left[H_{n}\right]
\end{array}\right] .
$$

Suppose the perturbative quantum system is controlled to track the desired reference state $r(t)=x_{d}$ by minimizing the following regulation cost function for stochastic optimal tracking control

$$
J=E\left[\eta_{f}{ }^{T} F \eta_{f}+\int_{0}^{t_{f}}\left(\eta^{T} Q \eta+\sum_{k=1}^{m} \rho_{k} u_{k}^{2}\right) d t\right]
$$

where $E$ denotes the operation of expectation.

Then we have the following result for stochastic quantum mechanical systems.

Theorem 2: The optimal tracking control for quantum mechanical system with stochastic perturbations in (14) is given by

$$
u_{k}^{o}=-\frac{1}{2 \rho_{k}} \frac{\partial V^{T}}{\partial \eta} B_{k} \eta, k=1,2, \ldots ., m
$$

in which $V(\eta, t)>0$ is obtained by solving the following HJE

$$
\begin{aligned}
& -\frac{\partial V}{\partial t}=\eta^{T} Q \eta+\frac{\partial V^{T}}{\partial \eta} A \eta-\sum_{k=1}^{m} \frac{1}{4 \rho_{k}} \frac{\partial V^{T}}{\partial \eta} B_{k} \eta \eta^{T} B_{k}^{T} \frac{\partial V}{\partial \eta} \\
& +\frac{1}{2} \eta^{T} C^{T} \frac{\partial^{2} V(\eta, t)}{\partial \eta^{2}} C \eta \\
& V\left(\eta_{f}, t_{f}\right)=\eta_{f}^{T} F \eta_{f}
\end{aligned}
$$

Proof: See Appendix 2.

From the analysis above, we need to solve the HJE (18) before we design the optimal tracking control (17) for perturbative quantum system (15). Therefore, the most important work to treat the optimal tracking control problem in the quantum mechanical system is to solve the HJE (13) or (18) at first. However, it is difficult to find a closed-form solution for (13) or (18). In this study, for the convenience of design, the techniques of tensor formal power series will be used to solve HJE (13) or (18) from the approximate perspective.

Remark 1: The environmental perturbation in quantum systems implies a dissipative behavior called decoherence. The optimal feedback control of such kind of quantum systems has been studied in [27]. Since this kind of model is more complicated than the model (14) [35], we leave it for further research. The model (14) can be used to deal with the control of one particle whose state can be represented by a state vector and is unnecessarily characterized by a density matrix. 


\section{OPTIMAL TRACKING CONTROL VIA TENSOR FORMAL POWER SERIES}

Due to the difficulty of obtaining the closed-form solution for the HJE (13), the techniques of tensor formal power series will be employed to solve the nonlinear partial differential HJE from the approximation point of view. At the beginning, the formulas about tensor and formal power series in $[25,36]$ will be introduced.

\subsection{Tensor Formal Power Series Approach}

The i-th order tensor in the $n$-dimensional space is a mathematical object that has $\mathrm{i}$ indices and $n^{i}$ components and obeys certain transformation rules. Here the tensor spaces are defined by the tensor product [36] of vectors.

Because the basic properties of tensors are similar to the vectors, we can write the tensors as the vector forms. So we will use kronecker product to indicate any tensor product below. For example, consider two vectors in 2-dimensional space $x=\left[\begin{array}{l}x_{1} \\ x_{2}\end{array}\right], y=\left[\begin{array}{l}y_{1} \\ y_{2}\end{array}\right]$, then the tensor product of them is $x \otimes y=\left[\begin{array}{l}x_{1} y_{1} \\ x_{1} y_{2} \\ x_{2} y_{1} \\ x_{2} y_{2}\end{array}\right]$. After that, we need to find the form of the linear operators on tensor space.

Remark 2: Let $\left\{e_{k} \mid k=1,2 \ldots n\right\}$ denote the basis of vectors in $R^{n}$, and $\left\{e_{k_{1}} \otimes e_{k_{2}} \otimes \ldots \otimes e_{k_{i}} \mid k_{j}=1,2, . ., n\right.$; $j=1,2, . ., i\}$ denote the basis of tensors in $\otimes_{i} R^{n}$. Since $\otimes_{i} R^{n}$ is a Hilbert space, we can consider linear operators defined on $\otimes_{i} R^{n}$. Let $\mathcal{L}\left(\otimes_{i} R^{n}\right)$ be the space of linear operators defined on $\otimes_{i} R^{n}$. We can define a linear operator $\mathbf{P}$ which has the relation [36]

$$
\mathbf{P}\left(e_{k_{1}} \otimes e_{k_{2}} \otimes \ldots \otimes e_{k_{i}}\right)=\sum_{\substack{k_{j}=1 \\ j=1,2, \ldots, i}}^{n} \mathbf{P}_{k_{1}, k_{2}, \ldots, k_{i}}^{l_{1}, l_{1}, \ldots, l_{i}}\left(e_{l_{1}} \otimes e_{l_{2}} \otimes \ldots \otimes e_{l_{i}}\right)
$$

here $\mathbf{P}_{k_{1}, k_{2}, \ldots, k_{i}}^{l_{1}, l_{2}, \ldots, l_{i}}$ is the corresponding element of the operator $\mathbf{P}$.

Now the inner product of two tensors $\left(x_{1} \otimes \ldots \otimes x_{i}\right)$ and $\left(y_{1} \otimes \ldots \otimes y_{i}\right)$ is defined as follows

$\left\langle\left(x_{1} \otimes \ldots . \otimes x_{i}\right),\left(y_{1} \otimes \ldots . \otimes y_{i}\right)\right\rangle$

$=\prod_{j=1}^{i}\left\langle x_{j}, y_{j}\right\rangle=\prod_{j=1}^{i} x_{j}^{T} y_{j}$

And

$\left\langle e_{k_{1}} \otimes e_{k_{2}} \otimes \ldots \otimes e_{k_{i}}, \quad e_{l_{1}} \otimes e_{l_{2}} \otimes \ldots \otimes e_{l_{i}}\right\rangle$

$=\left\{\begin{array}{rr}1 & \text { if } k_{h}=l_{h} \forall h \\ 0 & \text { otherwise }\end{array}\right.$
Then we define the adjoint $\mathbf{P}^{*}$ of $\mathbf{P}$ with the relation

$$
\begin{aligned}
\left\langle\mathbf{P}^{*}\left(v_{1} \otimes \ldots . \otimes v_{i}\right),\right. & \left.\left(u_{1} \otimes \ldots . \otimes u_{i}\right)\right\rangle= \\
& \left\langle\left(v_{1} \otimes \ldots \ldots \otimes v_{i}\right), \quad \mathbf{P}\left(u_{1} \otimes \ldots . \otimes u_{i}\right)\right\rangle
\end{aligned}
$$

From equation (20), $\mathbf{P}$ is self-adjoint if

$\mathbf{P}_{k_{1}, k_{2}, \ldots, k_{i}}^{l_{1}, l_{2}, \ldots, l_{i}}=\mathbf{P}_{l_{1}, l_{2}, \ldots, l_{i}}^{k_{1}, k_{2}, \ldots, k_{i}}$

If we deal with the tensor in the vector form, and use the kronecker product to indicate the tensor product, linear operators on the tensor space can be represented as matrices, and self-adjoint operators become symmetric matrices. If we set $v_{1}, \ldots, v_{i}, \quad w_{1}, \ldots, w_{i} \in R^{n}$, then two tensors $\left(v_{1} \otimes \ldots \otimes v_{i}\right)$ and $\left(w_{1} \otimes \ldots \otimes w_{i}\right)$ could be reformed to two corresponding vectors with $n^{i}$ elements, and the operating matrix on them is an $\left(n^{i}\right) \times\left(n^{i}\right)$ matrix, i.e., the matrix $\mathbf{P}$ has the element $\mathbf{P}_{k_{1}, k_{2}, \ldots, k_{i}}^{l_{1}, l_{2}, \ldots, l_{i}}$ in the $\left(1+\left(k_{1}-1\right) n^{i-1}+\left(k_{2}-1\right) n^{i-2}+\ldots+\left(k_{i}-1\right)\right)$-th column and the $\left(1+\left(l_{1}-1\right) n^{i-1}+\left(l_{2}-1\right) n^{i-2}+\ldots+\left(l_{i}-1\right)\right)$-th row.

For example, consider two vectors $x=\left[\begin{array}{l}x_{1} \\ x_{2}\end{array}\right], y=\left[\begin{array}{l}y_{1} \\ y_{2}\end{array}\right]$, then the equation $(y \otimes y)=\mathrm{P}(x \otimes x)$ can be rewritten in vector form as follows

$$
\left[\begin{array}{l}
y_{1} y_{1} \\
y_{1} y_{2} \\
y_{2} y_{1} \\
y_{2} y_{2}
\end{array}\right]=\left[\begin{array}{llll}
P_{1,1}^{1,1} & P_{1,2}^{1,1} & P_{2,1}^{1,1} & P_{2,2}^{1,1} \\
P_{11}^{1,2} & P_{1,2}^{1,2} & P_{2,1}^{1,2} & P_{2,2}^{1,2} \\
P_{1,1}^{2,1} & P_{1,2}^{2,1} & P_{2,1}^{2,1} & P_{2,2}^{2,1} \\
P_{1,1}^{2,2} & P_{1,2}^{2,2} & P_{2,1}^{2,2} & P_{2,2}^{2,2}
\end{array}\right]\left[\begin{array}{l}
x_{1} x_{1} \\
x_{1} x_{2} \\
x_{2} x_{1} \\
x_{2} x_{2}
\end{array}\right]
$$

Lemma 1 [3]: We note $\otimes_{i} x$ is an $\left(n^{i}\right) \times 1$ vector with the $i$ times kronecker product of $x$, and the operating matrices $P, Q \in R^{\left(n^{i}\right) \times\left(n^{i}\right)}$.

Then

$$
\begin{aligned}
& \text { (i) }\left(\frac{\partial\left(\left(\otimes_{i} x\right)^{T} P\left(\bigotimes_{i} x\right)\right)}{\partial x}\right)^{T} x=2 i\left(\left(\otimes_{i} x\right)^{T} P\left(\bigotimes_{i} x\right)\right) \\
& \text { (ii) }\left(\frac{\partial\left(\left(\bigotimes_{i} x\right)^{T} P\left(\bigotimes_{i} x\right)\right)}{\partial x}\right)^{T} x=2 i\left(\left(\bigotimes_{i} x\right)^{T} P\left(\otimes_{i} x\right)\right) \\
& \left(\left(\otimes_{i+j} x\right)^{T}(P \otimes Q)\left(\otimes_{i+j} x\right)\right)
\end{aligned}
$$

here $P$ is symmetric, and $(P \otimes Q)$ means a kronecker product.

Lemma 2: To generalize equation (22), it becomes

$$
\left(\frac{\partial\left(\left(\otimes_{i} x\right)^{T} P\left(\otimes_{i} x\right)\right)}{\partial x}\right)^{T} C x=2\left(\left(\otimes_{i} x\right)^{T} P \phi_{i}(C)\left(\otimes_{i} x\right)\right)
$$


here $P \in \mathcal{L}\left(\otimes_{i} R^{n}\right)$ and $P$ can be represented as an $\left(n^{i}\right) \times\left(n^{i}\right)$ symmetric matrix, $C$ is an $n \times n$ matrix, and $\phi_{i}(C)$ is an $\left(n^{i}\right) \times\left(n^{i}\right)$ matrix related to the matrix $C$. The element $\left(\phi_{i}(C)\right)_{k_{1}, k_{2}, \ldots, k_{i}}^{l_{1}, l_{2}, \ldots, l_{i}}$ of the matrix $\phi_{i}(C)$ satisfies

$$
\begin{aligned}
& \left(\phi_{i}(C)\right)_{k_{1}, k_{2}, \ldots, k_{i}}^{l_{1}, l_{2}, l_{i}} \\
& =\left\{\begin{array}{cc}
C_{k_{1}, k_{1}}+C_{k_{2}, k_{2}}+\ldots+C_{k_{i}, k_{i}} & \text { if } l_{1}=k_{1}, l_{2}=k_{2}, \ldots \ldots, \\
C_{l_{j}, k_{j}} & \text { if } l_{1}=k_{1}, \ldots l_{j-1}=k_{j-1}, \\
& l_{j+1}=k_{j+1}, \ldots, l_{i}=k_{i}, \text { but }\left(l_{j} \neq k_{j}\right) \\
0 & \text { others }
\end{array}\right.
\end{aligned}
$$

The detailed analyses about Lemma 2 and $\phi_{i}(C)$ are given in Appendix 3.

Lemma 3: Consider the following operation equation

$$
x^{T} C^{T} \frac{\partial^{2}\left(\left(\otimes_{i} x\right)^{T} P\left(\bigotimes_{i} x\right)\right)}{\partial x^{2}} C x=2\left(\left(\otimes_{i} x\right)^{T} R_{i}\left(\bigotimes_{i} x\right)\right)
$$

then the matrix $R_{i}$ could be represented as follows

$$
R_{i}=\phi_{i}(C)^{T} P \phi_{i}(C)+P \phi_{i}(C)^{2}-P \phi_{i}\left(C^{2}\right)
$$

where the matrix $\phi_{i}(C)$ is the same as (24), and $P$ is symmetric. 4.

The detailed derivation of Lemma 3 is given in Appendix

The calculation techniques of tensor power series approximation that we need to solve (13) and (18) are ready as shown above. Now we use these techniques to handle the quantum tracking control design problem.

We set the solution $V(\eta, t)$ of the HJE in (13) or (18) to be of the tensor formal power series form

$$
V(\eta, t)=\sum_{i=1}^{\infty}\left(\otimes_{i} \eta\right)^{T} P_{i}(t)\left(\otimes_{i} \eta\right)
$$

where $\eta \in R^{4 n}$ and $P_{i}(t)$ are continuous, time-varying $(4 n)^{i} \times(4 n)^{i}$ symmetric matrices. Before using the tensor power series to solve the HJE in (13) and (18), some related derivations should be given at first. Substituting (28) into (13) with the help of (23) and (24), we obtain

$$
\begin{aligned}
& \frac{\partial V}{\partial t}=\sum_{i=1}^{\infty}\left(\otimes_{i} \eta\right)^{T} \dot{P}_{i}(t)\left(\otimes_{i} \eta\right) \\
& \frac{\partial V^{T}}{\partial \eta} A \eta=2 \sum_{i=1}^{\infty}\left(\otimes_{i} \eta\right)^{T} P_{i}(t) \phi_{i}(A)\left(\otimes_{i} \eta\right) \\
& \sum_{k=1}^{m} \frac{1}{4 \rho_{k}} \frac{\partial V^{T}}{\partial \eta} B_{k} \eta \eta^{T} B_{k}^{T} \frac{\partial V}{\partial \eta} \\
& \quad=\sum_{k=1}^{m} \frac{1}{4 \rho_{k}}\left(\left(2 \sum_{r=1}^{\infty}\left(\otimes_{r} \eta\right)^{T} P_{r}(t) \phi_{r}\left(B_{k}\right)\left(\otimes_{r} \eta\right)\right)\right.
\end{aligned}
$$

$$
\begin{array}{r}
\left.\left(2 \sum_{s=1}^{\infty}\left(\otimes_{s} \eta\right)^{T} P_{s}(t) \phi_{s}\left(B_{k}\right)\left(\otimes_{s} \eta\right)\right)\right) \\
=\sum_{k=1}^{m} \frac{1}{\rho_{k}}\left(\sum_{r=1}^{\infty} \sum_{s=1}^{\infty}\left(\otimes_{r+s} \eta\right)^{T}\left(P_{r}(t) \phi_{r}\left(B_{k}\right)\right)\right. \\
\left.\otimes\left(P_{s}(t) \phi_{s}\left(B_{k}\right)\right)\left(\otimes_{r+s} \eta\right)\right)
\end{array}
$$

Therefore, from (29), (13) becomes

$$
\begin{gathered}
-\sum_{i=1}^{\infty}\left(\otimes_{i} \eta\right)^{T} \dot{P}_{i}(t)\left(\otimes_{i} \eta\right)= \\
\eta^{T} Q \eta+2 \sum_{i=1}^{\infty}\left(\otimes_{i} \eta\right)^{T} P_{i}(t) \phi_{i}(A)\left(\otimes_{i} \eta\right) \\
-\sum_{k=1}^{m} \frac{1}{\rho_{k}}\left(\sum_{r=1}^{\infty} \sum_{s=1}^{\infty}\left(\otimes_{r+s} \eta\right)^{T}\left(P_{r}(t) \phi_{r}\left(B_{k}\right)\right)\right. \\
\left.\otimes\left(P_{s}(t) \phi_{s}\left(B_{k}\right)\right)\left(\otimes_{r+s} \eta\right)\right)
\end{gathered}
$$

with the terminal condition

$$
V\left(\eta_{f}, t_{f}\right)=\eta_{f}^{T} F \eta_{f}=\sum_{i=1}^{\infty}\left(\otimes_{i} \eta_{f}\right)^{T} P_{i}\left(t_{f}\right)\left(\otimes_{i} \eta_{f}\right)
$$

Now, equating like powers in (30) leads to the following Riccati-like equations

$$
\begin{aligned}
\dot{P}_{1}(t)+ & P_{1}(t) A+A^{T} P_{1}(t)+Q=0 \quad \text { for } i=1 \\
\dot{P}_{i}(t)+ & P_{i}(t) \phi_{i}(A)+\phi_{i}^{T}(A) P_{i}(t) \\
& -\sum_{k=1}^{m} \frac{1}{\rho_{k}}\left(\sum_{\substack{r+s=i \\
r, s \geq 1}}\left(P_{r}(t) \phi_{r}\left(B_{k}\right)\right) \otimes\left(P_{s}(t) \phi_{s}\left(B_{k}\right)\right)\right)=0
\end{aligned}
$$

for $i=2,3, \ldots$

with terminal conditions

$P_{1}\left(t_{f}\right)=F, \quad P_{i}\left(t_{f}\right)=0, i=2,3, \ldots \ldots$

where the calculations of $\phi_{i}(A), \phi_{i}\left(B_{k}\right), i=2,3, \ldots \ldots$ are given in (25). After solving $P_{i}(t)$ from the Riccati-like differential equation in (32), we get the optimal tracking control from (12) as follows

$$
u_{k}^{o}=-\frac{1}{\rho_{k}} \sum_{i}\left(\otimes_{i} \eta\right)^{T} P_{i} \phi_{i}\left(B_{k}\right)\left(\otimes_{i} \eta\right), k=1,2, \ldots ., m
$$

By induction, we can rewrite (32b) as

$$
\begin{aligned}
& \dot{P}_{i}(t)+P_{i}(t) \phi_{i}(A)+\phi_{i}^{T}(A) P_{i}(t)- \\
& \sum_{k=1}^{m} \frac{1}{2 \rho_{k}}\left(\sum _ { \substack { r + s = i \\
r , s \geq 1 } } \left\{\left(\left(\phi_{r}\left(B_{k}\right)\right)^{T}\left(P_{r}(t)\right)\right) \otimes\left(\left(\phi_{s}\left(B_{k}\right)\right)^{T}\left(P_{s}(t)\right)\right)\right.\right. \\
& \left.+\left(P_{r}(t) \phi_{r}\left(B_{k}\right)\right) \otimes\left(P_{s}(t) \phi_{s}\left(B_{k}\right)\right)\right\}=0
\end{aligned}
$$

and so $P_{i}(t)$ is symmetric. 


\subsection{Stochastic Tracking Control Design}

Now we further investigate the optimal tracking control of a stochastic quantum system in (14). After substituting (28) into (18) with the help of (23), (24), (26), and (27), we could also solve this HJE. The additional term in (18) should be considered as

$$
\begin{aligned}
& \frac{\partial^{2} V^{T}}{\partial \eta^{2}} C \eta=\sum_{i=1}^{\infty}\left(\left(\otimes_{i} \eta\right)^{T}\right. \\
& \left.\left(\phi_{i}(C)^{T} P_{i}(t) \phi_{i}(C)+P_{i}(t) \phi_{i}(C)^{2}-P_{i}(t) \phi_{i}\left(C^{2}\right)\right)\left(\otimes_{i} \eta\right)\right)
\end{aligned}
$$

and thus the HJE in (18) becomes

$$
\begin{aligned}
& -\sum_{i=1}^{\infty}\left(\otimes_{i} \eta\right)^{T} \dot{P}_{i}(t)\left(\otimes_{i} \eta\right) \\
& =\eta^{T} Q \eta+2 \sum_{i=1}^{\infty}\left(\otimes_{i} \eta\right)^{T} P_{i}(t) \phi_{i}(A)\left(\otimes_{i} \eta\right) \\
& -\sum_{k=1}^{m} \frac{1}{\rho_{k}}\left[\sum_{r=1}^{\infty} \sum_{s=1}^{\infty}\left(\otimes_{r+s} \eta\right)^{T}\left(P_{r}(t) \phi_{r}\left(B_{k}\right)\right)\right. \\
& \left.\qquad\left(P_{s}(t) \phi_{s}\left(B_{k}\right)\right)\left(\otimes_{r+s} \eta\right)\right] \\
& +\sum_{i=1}^{\infty}\left[\left(\otimes_{i} \eta\right)^{T}\right. \\
& \left.\left(\phi_{i}(C)^{T} P_{i}(t) \phi_{i}(C)+P_{i}(t) \phi_{i}(C)^{2}-P_{i}(t) \phi_{i}\left(C^{2}\right)\right)\left(\otimes_{i} \eta\right)\right]
\end{aligned}
$$

with the terminal condition

$$
V\left(\eta_{f}, t_{f}\right)=\eta_{f}^{T} F \eta_{f}=\sum_{i=1}^{\infty}\left(\otimes_{i} \eta_{f}\right)^{T} P_{i}\left(t_{f}\right)\left(\otimes_{i} \eta_{f}\right)
$$

Now, equating like powers in (36) leads to the following Riccati-like equations

$$
\begin{gathered}
\dot{P}_{1}(t)+P_{1}(t) A+A^{T} P_{1}(t)+Q+C^{T} P_{1}(t) C=0 \text { for } i=1 \\
\dot{P}_{i}(t)+P_{i}(t) \phi_{i}(A)+\phi_{i}^{T}(A) P_{i}(t) \\
-\sum_{k=1}^{m} \frac{1}{\rho_{k}}\left(\sum_{r+s=i}\left(P_{r}(t) \phi_{r}\left(B_{k}\right)\right) \otimes\left(P_{s}(t) \phi_{s}\left(B_{k}\right)\right)\right) \\
+\left(\phi_{i}(C)^{T} P_{i}(t) \phi_{i}(C)+P_{i}(t) \phi_{i}(C)^{2}-P_{i}(t) \phi_{i}\left(C^{2}\right)\right)=0, \\
i=2,3, \ldots
\end{gathered}
$$

with the terminal conditions

$$
P_{1}\left(t_{f}\right)=F, \quad P_{i}\left(t_{f}\right)=0 \text { for } i=2,3, \ldots \ldots
$$

After solving the Riccati-like equations in (13) to obtain $P_{1}(t), P_{2}(t), \ldots, P_{i}(t), \ldots$, the optimal tracking controls in $(17)$ for perturbative quantum system (14) become

$$
u_{k}^{o}=-\frac{1}{\rho_{k}} \sum_{i}\left(\otimes_{i} \eta\right)^{T} P_{i} \phi_{i}\left(B_{k}\right)\left(\otimes_{i} \eta\right), k=1,2, \ldots ., m
$$

In general, the tensor formal series have infinite terms to be solved. However, it is difficult to implement high-order terms $P_{i}(t)$ due to their complicated computation. In this study, only a few important terms of $P_{i}(t)$ are considered to approximate the optimal tracking design. A two-level spin system and a three-level quantum system are both discussed below to illustrate the design procedure of the proposed tracking control.

\subsection{Comparisons with Other Approaches}

Now we provide a detailed comparative analysis of the proposed scheme with the existing schemes [4-7,26-28].

Our proposed scheme has the following advantages: First, our control approach can track any desired reference state, whereas the conventional optimal quantum control in [4-7, $26,28]$ only can regulate the quantum system to a fixed quantum system state but can not track any desired reference state. Second, the proposed control strategy can minimize the terminal tracking error, the tracking path error and the control effort simultaneously. By contrast, only the total control energy is minimized in [26], only both the total control energy and the terminal state error are minimized in $[4,5,7,28]$ and the total control energy, the terminal state error and the state energy are minimized in $[6,27]$. Therefore, those schemes in [4-7, 26-28] are not suitable for tracking design of quantum systems, since their control strategies are not to minimize the tracking path error. Third, a tensor formal power series approach is employed to solve the HJE approximately since it is still not easy to solve an HJE analytically, whereas there is no method concerning solving an HJE in [27] although its optimal control is also related to an HJE. Fourth, our control approach can be suitable for an optimal stochastic tracking control while the parameter variation noise and modeling error exist, whereas $[4,6,7,26,28]$ can not deal with the stochastic optimal control of quantum systems.

But, our proposed scheme has one disadvantage that is somewhat much computational complexity due to a tensor formal power series. However, our numerical computation time is much reduced since there is no iterative procedure to search the optimal control. By contrast, there is much computational complexity in [5-7, 27-28] due to an inextricable iterative procedure to solve two coupled differential equations, i.e. the state and costate equations, and then update the optimal control at each iteration step. Since [4-7, 26-28] are not suitable for tracking design of quantum systems, the controllers are quite different from ours so that comparisons of the computational complexity of their schemes to ours are not easily discussed. Moreover, there is no method concerning solving the HJE in [27], the comparison of the computational complexity of their scheme to ours is not easy to discuss either.

\section{SIMULATION EXAMPLES}

In this section, several simulation examples of two-level and three-level quantum systems are given to illustrate the design procedure of the optimal tracking control by the tensor formal power series method. Two-level Quantum systems are the most basic quantum systems with important applications, especially in communications and computation [26]. A two-level quantum system means a system which has two energy levels (in other words, the system has two eigen- 
values and two eigenstates). A two-level system is used to generate a sequence of desired quantum bits for quantum computing and data communications [26], which could be formulated as a tracking control problem of quantum system. Some of the systems have the property that they have two close levels far away from others, and can be approximated by the two-level systems. The spin $1 / 2$ systems are two-level systems and have been studied extensively. Here we consider a state tracking control problem of a spin $1 / 2$ atomic nucleus [26].

A two-level-spin particle (here it means an atomic nucleus) is controlled by an external magnetic field similar to the one in the nuclear magnetic resonance (NMR) experiments [26]. First we fix magnetic field $B_{z}$ in the $z$ direction, and the fixed field determines the direction of spin and decides the eigenstates of the spin. The magnetic field in the $x-y$ direction is varied to change or control the direction of spin. The varied field can be controlled by two orthogonal components $u_{x}$ and $u_{y}$, or one of them. The system is described in Fig. (2). Here the control purpose is to steer the spin from one eigenstate to a desired state to form a quantum logic gate in quantum computing [26, 31,34], and the initial state could be prepared by Stern-Gerlach apparatus [37].

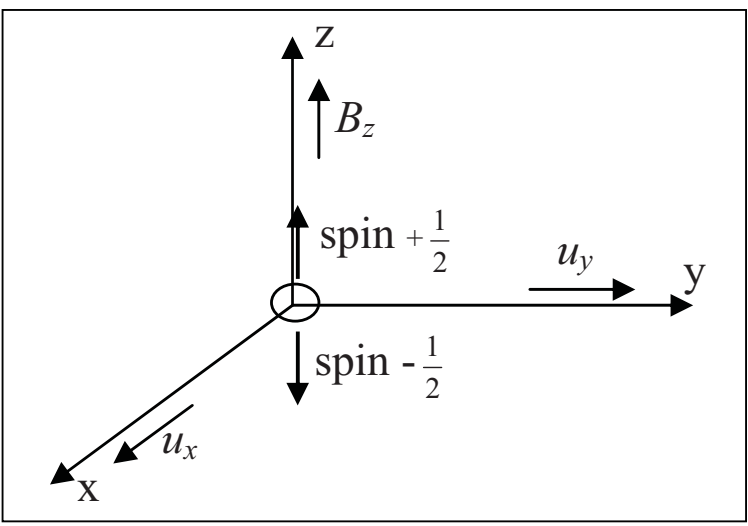

Fig. (2). Two-level spin control system.

Considering the control of a spin $1 / 2$ nucleus, the dynamic equation of the spin system is derived in some text books of quantum mechanics [29]. The quantum dynamic equation is given as follows

$$
\begin{aligned}
& i \hbar \dot{\psi}(t)= \\
& \left(-\gamma B_{z} \frac{\hbar}{2}\left[\begin{array}{cc}
1 & 0 \\
0 & -1
\end{array}\right]-\gamma u_{x} \frac{\hbar}{2}\left[\begin{array}{ll}
0 & 1 \\
1 & 0
\end{array}\right]-\gamma u_{y} \frac{\hbar}{2}\left[\begin{array}{cc}
0 & -i \\
i & 0
\end{array}\right]\right) \psi(t)
\end{aligned}
$$

where $\gamma$ is the gyromagnetic ratio associated with the spin system, $B_{z}$ is the fixed component of the magnetic field and $u_{x}, u_{y}$ are the components of the magnetic fields varied with the tracking control laws. The spin $1 / 2$ system has eigenstates $\psi_{\uparrow}:=\left[\begin{array}{l}1 \\ 0\end{array}\right], \psi_{\downarrow}:=\left[\begin{array}{l}0 \\ 1\end{array}\right]$.
By rescaling $t \rightarrow\left(-\frac{\gamma B_{z} t}{2}\right), \psi(t)$ becomes $\psi\left(-\frac{\gamma B_{z} t}{2}\right)$, $u_{x}(t)$ becomes $u_{x}\left(-\frac{\gamma B_{z} t}{2}\right), \quad$ and $u_{y}(t)$ becomes $u_{y}\left(-\frac{\gamma B_{z} t}{2}\right)$. Equation (39) can be rewritten as

$i \psi(t)=\left(\left[\begin{array}{cc}1 & 0 \\ 0 & -1\end{array}\right]+\frac{u_{x}}{B_{z}}\left[\begin{array}{ll}0 & 1 \\ 1 & 0\end{array}\right]+\frac{u_{y}}{B_{z}}\left[\begin{array}{cc}0 & -i \\ i & 0\end{array}\right]\right) \psi(t)$

And further rescaling $u_{x} \rightarrow\left(\frac{u_{x}}{B_{z}}\right), u_{y} \rightarrow\left(\frac{u_{y}}{B_{z}}\right)$, equation (40) can be rewritten as

$i \dot{\psi}(t)=\left(\left[\begin{array}{cc}1 & 0 \\ 0 & -1\end{array}\right]+u_{x}\left[\begin{array}{ll}0 & 1 \\ 1 & 0\end{array}\right]+u_{y}\left[\begin{array}{cc}0 & -i \\ i & 0\end{array}\right]\right) \psi(t)$

For common NMR devices, we set the fixed magnetic field $B_{z}$ as -4.69 Tesla (for example: in the NMR device, Bruker AC 200, it makes the hydrogen nucleus with the resonance frequency $200 \mathrm{MHz}$ ). By controlling the hydrogen nucleus with the gyromagnetic ratio $\gamma=2.6751 \times 10^{8} \mathrm{rad} / \mathrm{s}-$ Tesla $=42.58 \mathrm{MHz} /$ Tesla, the time of the simulated result in (41) can be scaled with $\frac{2}{\gamma B_{z}}=10^{-8}$, and the control magnetic field will be scaled with -4.69 .

In this design example, three tracking control cases are considered with one control input, two control inputs, and one control input with stochastic parameter perturbation, respectively.

Example 1: Spin system tracking with one control input

First we consider the spin system controlled by a magnetic field in the $y$-direction. The dynamic equation of the controlled spin system is given by

$i \dot{\psi}(t)=\left(\left[\begin{array}{cc}1 & 0 \\ 0 & -1\end{array}\right]+u_{1}(t)\left[\begin{array}{cc}0 & -i \\ i & 0\end{array}\right]\right) \psi(t), \quad \psi(0)=\left[\begin{array}{l}1 \\ 0\end{array}\right]$

By (6), the corresponding state space of (42) can be rewritten as

$$
\dot{x}=G_{0} x+u_{1} G_{1} x, x(0)=x_{0}
$$

where

$G_{0}=\left[\begin{array}{cccc}0 & 0 & 1 & 0 \\ 0 & 0 & 0 & -1 \\ -1 & 0 & 0 & 0 \\ 0 & 1 & 0 & 0\end{array}\right], G_{1}=\left[\begin{array}{cccc}0 & -1 & 0 & 0 \\ 1 & 0 & 0 & 0 \\ 0 & 0 & 0 & -1 \\ 0 & 0 & 1 & 0\end{array}\right]$

and $x_{0}=\left[\begin{array}{llll}1 & 0 & 0 & 0\end{array}\right]^{T}$ corresponds to the spin-up state $\psi_{\uparrow}:=\left[\begin{array}{l}1 \\ 0\end{array}\right]$.

And the reference model for the desired state is given by

$$
\dot{r}=0, \quad r(0)=r_{0}=x_{d}
$$


where $x_{d}=\left[\begin{array}{llll}0 & 1 & 0 & 0\end{array}\right]^{T}$ corresponds to the spin-down state $\psi_{\downarrow}:=\left[\begin{array}{l}0 \\ 1\end{array}\right]$.

Therefore, we steer the spin from the spin-up state $x_{0}$ to the spin-down state $x_{d}$.

For the tracking cost in (8), we specify

$$
F_{0}=Q_{0}=\left[\begin{array}{llll}
1 & 0 & 0 & 0 \\
0 & 1 & 0 & 0 \\
0 & 0 & 1 & 0 \\
0 & 0 & 0 & 1
\end{array}\right], \rho_{1}=4, t_{f}=10
$$

where $t_{f}=10 * 10^{-8} s=10^{-7} \mathrm{~s}$ after recovering the scale of real quantum system.

After regulating, we have the augmented quantum system (10) and the corresponding optimal control cost function (11). The matrices in (10) and (11) are shown as follows.

$$
\begin{aligned}
& A=\left[\begin{array}{cccccccc}
0 & 0 & 1 & 0 & 0 & 0 & 0 & 0 \\
0 & 0 & 0 & -1 & 0 & 0 & 0 & 0 \\
-1 & 0 & 0 & 0 & 0 & 0 & 0 & 0 \\
0 & 1 & 0 & 0 & 0 & 0 & 0 & 0 \\
0 & 0 & 0 & 0 & 0 & 0 & 0 & 0 \\
0 & 0 & 0 & 0 & 0 & 0 & 0 & 0 \\
0 & 0 & 0 & 0 & 0 & 0 & 0 & 0 \\
0 & 0 & 0 & 0 & 0 & 0 & 0 & 0
\end{array}\right], \\
& B_{1}=\left[\begin{array}{cccccccc}
0 & -1 & 0 & 0 & 0 & 0 & 0 & 0 \\
1 & 0 & 0 & 0 & 0 & 0 & 0 & 0 \\
0 & 0 & 0 & -1 & 0 & 0 & 0 & 0 \\
0 & 0 & 1 & 0 & 0 & 0 & 0 & 0 \\
0 & 0 & 0 & 0 & 0 & 0 & 0 & 0 \\
0 & 0 & 0 & 0 & 0 & 0 & 0 & 0 \\
0 & 0 & 0 & 0 & 0 & 0 & 0 & 0 \\
0 & 0 & 0 & 0 & 0 & 0 & 0 & 0
\end{array}\right], \\
& F=Q=\left[\begin{array}{cccccccc}
1 & 0 & 0 & 0 & -1 & 0 & 0 & 0 \\
0 & 1 & 0 & 0 & 0 & -1 & 0 & 0 \\
0 & 0 & 1 & 0 & 0 & 0 & -1 & 0 \\
0 & 0 & 0 & 1 & 0 & 0 & 0 & -1 \\
-1 & 0 & 0 & 0 & 1 & 0 & 0 & 0 \\
0 & -1 & 0 & 0 & 0 & 1 & 0 & 0 \\
0 & 0 & -1 & 0 & 0 & 0 & 1 & 0 \\
0 & 0 & 0 & -1 & 0 & 0 & 0 & 1
\end{array}\right]
\end{aligned}
$$

For the simplicity of computation, only $n_{a}=2$ is considered to approximate the control law. After recovering the scale, the simulation result is shown in Fig. (3). So the probability, i.e. $\left|c_{1}(t)\right|^{2}=x_{1}(t)^{2}+x_{3}(t)^{2}$, of the spin-up state and the probability, i.e. $\left|c_{2}(t)\right|^{2}=x_{2}(t)^{2}+x_{4}(t)^{2}$, of the spindown state are simulated in Fig. (3) to confirm the tracking performance of the proposed method. It is seen that the state of the two-level quantum system can track the desired state quite well with only one control input.

Because the Riccati-like equations (32) could be efficiently solved by the toolbox in Matlab, the proposed optimal tracking control in (33) by tensor formal power series could be easily calculated, especially for $n_{a}=2$ case in this example.
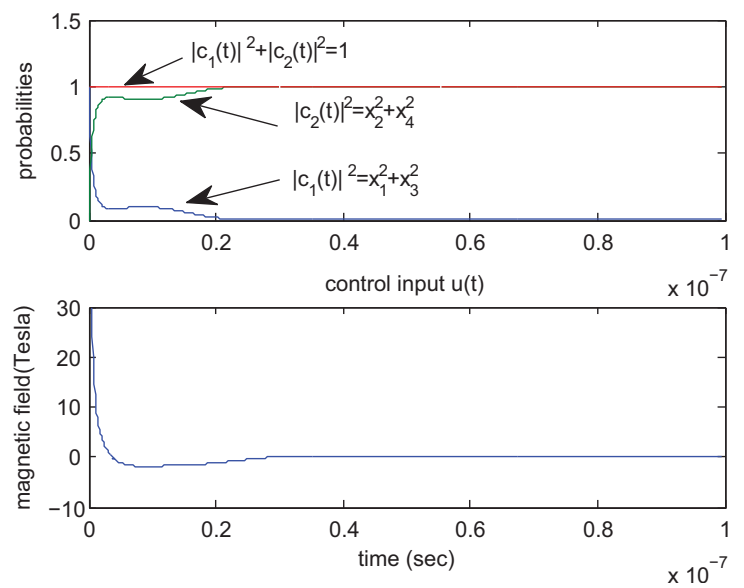

Fig. (3). The probabilities of eigenstates (upper half side), and the corresponding control signal (lower half side) for the state tracking control in the two-level quantum system by a magnetic field in the $y$-direction in example 1 .

Example 2: Spin system tracking control with two inputs

Suppose two control inputs with the magnetic fields in the $\mathrm{x}$-direction and $\mathrm{y}$-direction are employed to control this spin quantum system. The dynamic equation of the spin system with two control inputs is given by:

$$
\begin{aligned}
& i \dot{\psi}(t)=\left(\left[\begin{array}{cc}
1 & 0 \\
0 & -1
\end{array}\right]+u_{1}(t)\left[\begin{array}{ll}
0 & 1 \\
1 & 0
\end{array}\right]+u_{2}(t)\left[\begin{array}{cc}
0 & -i \\
i & 0
\end{array}\right]\right) \psi(t), \\
& \psi(0)=\left[\begin{array}{l}
1 \\
0
\end{array}\right]
\end{aligned}
$$

The initial value of $x$ in (7) is set as $x(0)=x_{0}=\left[\begin{array}{llll}1 & 0 & 0 & 0\end{array}\right]^{T}$.

Suppose we control the quantum state with a magnetic field to track the following desired quantum state

$x_{d}(t)=\left\{\begin{array}{l}{\left[\begin{array}{llll}0 & 1 & 0 & 0\end{array}\right]^{T} \text { as } \mathrm{t}=\left(0, \frac{\mathrm{t}_{\mathrm{f}}}{2}\right]} \\ {\left[\begin{array}{llll}1 & 0 & 0 & 0\end{array}\right]^{T} \text { as } \mathrm{t}=\left(\frac{\mathrm{t}_{\mathrm{f}}}{2}, \mathrm{t}_{\mathrm{f}}\right)}\end{array}\right.$

, i.e., the spin system is to be controlled from the spin-up state at time $\mathrm{t}=0$ to the spin-down state within $\left[0, \frac{\mathrm{t}_{\mathrm{f}}}{2}\right]$ and then back to the spin-up state within $\left[\frac{t_{f}}{2}, t_{f}\right]$.

Similar to the above example, the weightings in the tracking cost in (8) are given by 
$F_{0}=Q_{0}=\left[\begin{array}{llll}1 & 0 & 0 & 0 \\ 0 & 1 & 0 & 0 \\ 0 & 0 & 1 & 0 \\ 0 & 0 & 0 & 1\end{array}\right], \rho_{1}=\rho_{2}=5$

$t_{f}=10\left(\right.$ here $t_{f}=1 \times 10^{-7} \mathrm{~s}$ in real system $)$.

After regulation transformation, the matrices $A, F$, and $Q$ are the same as (45), and $B_{1}, B_{2}$ are given as follows

$$
\begin{aligned}
B_{1} & =\left[\begin{array}{cccccccc}
0 & 0 & 0 & 1 & 0 & 0 & 0 & 0 \\
0 & 0 & 1 & 0 & 0 & 0 & 0 & 0 \\
0 & -1 & 0 & 0 & 0 & 0 & 0 & 0 \\
-1 & 0 & 0 & 0 & 0 & 0 & 0 & 0 \\
0 & 0 & 0 & 0 & 0 & 0 & 0 & 0 \\
0 & 0 & 0 & 0 & 0 & 0 & 0 & 0 \\
0 & 0 & 0 & 0 & 0 & 0 & 0 & 0 \\
0 & 0 & 0 & 0 & 0 & 0 & 0 & 0
\end{array}\right], \\
B_{2} & =\left[\begin{array}{cccccccc}
0 & -1 & 0 & 0 & 0 & 0 & 0 & 0 \\
1 & 0 & 0 & 0 & 0 & 0 & 0 & 0 \\
0 & 0 & 0 & -1 & 0 & 0 & 0 & 0 \\
0 & 0 & 1 & 0 & 0 & 0 & 0 & 0 \\
0 & 0 & 0 & 0 & 0 & 0 & 0 & 0 \\
0 & 0 & 0 & 0 & 0 & 0 & 0 & 0 \\
0 & 0 & 0 & 0 & 0 & 0 & 0 & 0 \\
0 & 0 & 0 & 0 & 0 & 0 & 0 & 0
\end{array}\right],
\end{aligned}
$$

For the simplicity of computation, $n_{a}=2$ is considered. After recovering the scale, the simulation result is given in Fig. (4). It is seen that the tracking performance with two control inputs is much better than that with only one control input, especially in the transient response. This is because two control input has more degrees of freedom to manipulate the control efforts to track the desired reference sequence than one control input case.
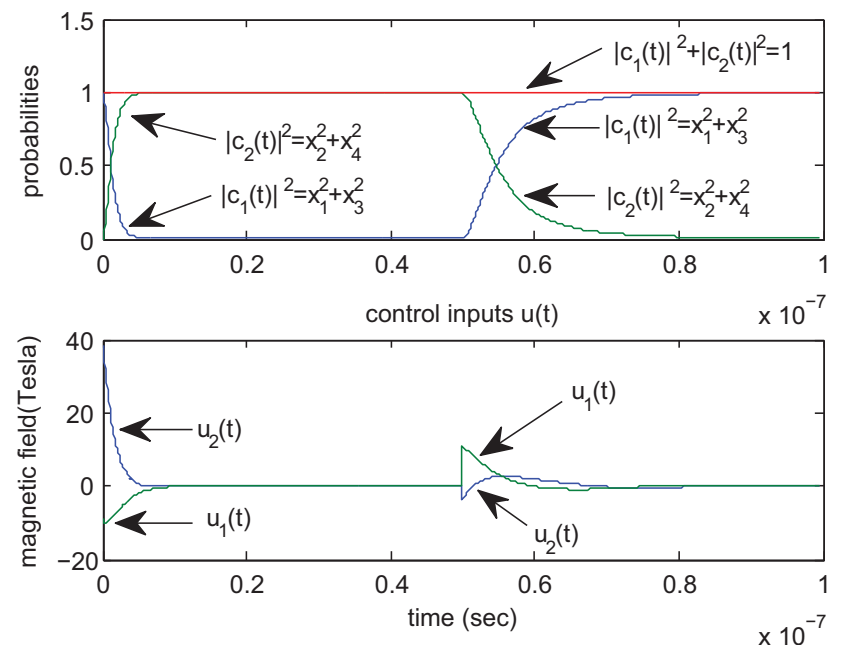

Fig. (4). The probabilities of eigenstates (upper half side), and the corresponding control signals (lower half side) for the system tracking control in the two-level quantum system by two magnetic fields in the $x$-direction and the $y$-direction, respectively, in example 2 .
Example 3: Spin tracking system with stochastic perturbations

Consider the following spin system with stochastic perturbations

$$
\begin{aligned}
i d \psi(t)= & {\left[\begin{array}{cc}
1 & 0 \\
0 & -1
\end{array}\right] \psi(t) d t+u_{1}(t)\left[\begin{array}{cc}
0 & -i \\
i & 0
\end{array}\right] \psi(t) d t } \\
& +\left[\begin{array}{cc}
0.1 & -0.1 i \\
0.1 i & -0.1
\end{array}\right] \psi(t) d W
\end{aligned}
$$

$\psi(0)=\left[\begin{array}{l}1 \\ 0\end{array}\right]$

The initial value of $x$ in (7) is set as $x(0)=x_{0}=\left[\begin{array}{llll}1 & 0 & 0 & 0\end{array}\right]^{T}$ and the reference model for the desired state is given by

$\dot{r}=0, r_{0}=x_{d}=\left[\begin{array}{llll}0 & 1 & 0 & 0\end{array}\right]^{T}$

After regulation transformation, we have the augmented quantum system and the corresponding optimal control cost function shown in (15) and (16). The matrices $A, B_{1}, F$, and $Q$ are the same as (45), and the other weightings in (15) and (16) are shown as follows

$$
C=\left[\begin{array}{cccccccc}
0 & -0.1 & 0.1 & 0 & 0 & 0 & 0 & 0 \\
0.1 & 0 & 0 & -0.1 & 0 & 0 & 0 & 0 \\
-0.1 & 0 & 0 & -0.1 & 0 & 0 & 0 & 0 \\
0 & 0.1 & 0.1 & 0 & 0 & 0 & 0 & 0 \\
0 & 0 & 0 & 0 & 0 & 0 & 0 & 0 \\
0 & 0 & 0 & 0 & 0 & 0 & 0 & 0 \\
0 & 0 & 0 & 0 & 0 & 0 & 0 & 0 \\
0 & 0 & 0 & 0 & 0 & 0 & 0 & 0
\end{array}\right],
$$

$\rho_{1}=4, t_{f}=10$ ( here $t_{f}=10^{-7} \mathrm{~s}$ in real system ).

For the simplicity of computation, $n_{a}=2$ is considered. After recovering the scale, the simulation result is shown in Fig. (5).
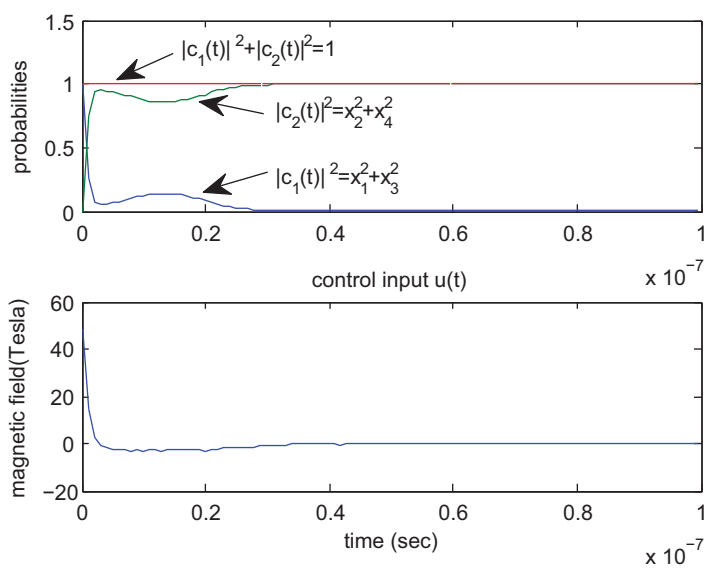

Fig. (5). The probabilities of eigenstates (upper half side), and the corresponding control signal (lower half side) for the stochastic system tracking control by a magnetic field in the $y$-direction in example 3. 
Because of stochastic perturbation, the tracking performance in this example is worse than that in Example 1. However, the tracking performance is still very satisfactory. Obviously, the proposed tracking control method is robust to the stochastic perturbation in quantum system and has much potential application to practical control designs.

Example 4: State tracking control for a three-level system

In order to confirm the proposed tracking control method to more complex quantum system, let us consider the following three-level quantum system [28]

$i \dot{\psi}(t)=H_{0} \psi(t)+u_{1}(t) H_{\text {tran }} \psi(t), \quad \psi(0)=\left[\begin{array}{l}0 \\ 1 \\ 0\end{array}\right]$.

where $H_{0}=\left[\begin{array}{ccc}1.0 & 0 & 0 \\ 0 & 2.0 & 0 \\ 0 & 0 & 3.0\end{array}\right]$ denotes the self Hamiltonian

of the three-level quantum system with each diagonal element representing the energy for each system level, $H_{\text {tran }}=\left[\begin{array}{lll}0 & 1 & 1 \\ 1 & 0 & 1 \\ 1 & 1 & 0\end{array}\right]$ denotes the electronic dipole transition matrix and $u_{1}(t)$ denotes the electric field which induces transitions between three levels of this quantum system.

Here we note that all calculations are carried out in atomic units (a.u.).

Suppose we control the quantum state to track the following desired quantum state

$\psi_{d}(t)=\left\{\begin{array}{l}{\left[\begin{array}{lll}1 & 0 & 0\end{array}\right]^{T} \text { as } \mathrm{t}=\left(0, \frac{\mathrm{t}_{\mathrm{f}}}{2}\right]} \\ {\left[\begin{array}{lll}0 & 0 & 1\end{array}\right]^{T} \text { as } \mathrm{t}=\left(\frac{\mathrm{t}_{\mathrm{f}}}{2}, \mathrm{t}_{\mathrm{f}}\right)}\end{array}\right.$

, i.e., the quantum system is to be controlled from the eigenstate 2 at time $\mathrm{t}=0$ to the eigenstate 1 within $\left[0, \frac{\mathrm{t}_{\mathrm{f}}}{2}\right]$ and then to the eigenstate 3 within $\left[\frac{\mathrm{t}_{\mathrm{f}}}{2}, \mathrm{t}_{\mathrm{f}}\right]$, where $t_{f}=30$ a.u.. The weighting matrices $F_{0}$ and $Q_{0}$ are the same as the ones in Example 1, and $\rho_{1}=25$. The simulation result is shown in Fig. (6).

Discussion: By the tensor power series to approximate the solution of the nonlinear HJE in (13), the optimal tracking control could be easily designed by solving the Riccatilike equations in (32), which could be easily solved with the help of toolbox in Matlab. Therefore the proposed method could simplify the design procedure even though the approximation procedure seems complicated. Further, in these examples we have tried other methods to solve the HJE of optimal tracking control design of quantum systems, e.g. fuzzy approximation technique, but the technique of tensor formal power series is simpler in design procedure and better in tracking performance. Further, the conventional quantum control design only control the system to an equilibrium state. But the proposed optimal tracking control design could make the quantum system track a sequence of desired states according to the reference model. Therefore, the proposed tracking method is more potential to generate a desired sequence of quantum bits for quantum communication and computation.
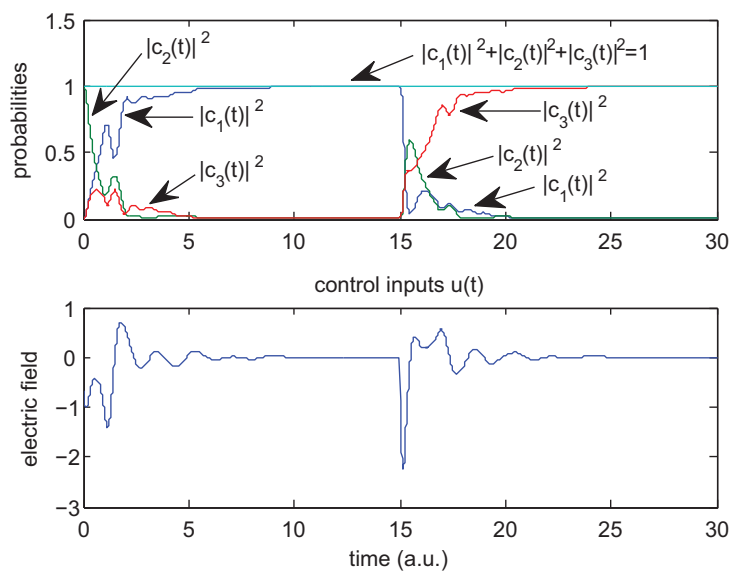

Fig. (6). The probabilities of eigenstates (upper half side), and the corresponding control signal (lower half side) for the state tracking control by an electric field in example 4 .

\section{CONCLUSIONS}

In this study, the state space model for quantum systems is constructed and then the optimal tracking control of quantum system is proposed to achieve any desired state based on Bellman dynamic programming method. In order to avoid the difficulty in solving the partial differential HJE equation directly, the techniques based on tensor formal power series is employed to obtain the approximate optimal tracking control law of quantum systems. The stochastic optimal tracking control design for quantum system with uncertain perturbation is also developed based on Itô-type differential equation to meet the practical noisy environment. Unlike the conventional optimal control is only to stabilize the quantum system to an equilibrium state, the proposed tracking method can track any desired state generated by a referenced model. Several simulation examples of two-level or three-level quantum systems are given to illustrate the design procedure of the optimal tracking control by the tensor formal power series method. Even with second order tensor power series approximation, the tracking performance is very satisfactory. It is also found that the tracking performance with twodirection control inputs is with shorter transient response than with one-direction control input. Even the design procedure is complicated because of the requirement of optimal tracking performance and approximating solution of HJE by tensor power series, the control law is very simple in the result. Therefore, the proposed optimal tracking control design is suitable to generate a sequence of desired states (or bits) with much potential application to quantum computation and communications.

\section{APPENDIX 1:}

Proof of Theorem 1: 
The optimal control in (10) and (11) is obtained by means of the solution $V(\eta, t)>0$ of the following Bellman dynamic programming equation [24]

$$
\begin{aligned}
& -\frac{\partial V(\eta, t)}{\partial t}= \\
& \min _{\substack{u_{k}(t) \\
k=1, \ldots, m}}\left\{\eta^{T} Q \eta+\frac{\partial V(\eta, t)^{T}}{\partial \eta}\left(A \eta+\sum_{k=1}^{m} u_{k} B_{k} \eta\right)+\sum_{k=1}^{m} \rho_{k} u_{k}^{2}\right\}
\end{aligned}
$$

with the terminal condition $V\left(\eta_{f}, t_{f}\right)=\eta_{f}^{T} F \eta_{f}$. Using the technique of completing of square, (49) becomes

$$
\begin{aligned}
-\frac{\partial V}{\partial t}=\min _{\substack{u_{k}(t) \\
k=1, \ldots, m}}\left\{\eta^{T} Q \eta+\frac{\partial V^{T}}{\partial \eta} A \eta+\right. & \\
\sum_{k=1}^{m}\left(\rho_{k} u_{k}\right. & \left.+\frac{1}{2} \eta^{T} B_{k}^{T} \frac{\partial V}{\partial \eta}\right)^{T} \frac{1}{\rho_{k}}\left(\rho_{k} u_{k}+\frac{1}{2} \eta^{T} B_{k}^{T} \frac{\partial V}{\partial \eta}\right) \\
& \left.-\sum_{k=1}^{m} \frac{1}{4 \rho_{k}} \frac{\partial V^{T}}{\partial \eta} B_{k} \eta \eta^{T} B_{k}^{T} \frac{\partial V}{\partial \eta}\right\}, \quad V\left(\eta_{f}, t_{f}\right)=\eta_{f}^{T} F \eta_{f}
\end{aligned}
$$

From the right hand side of (50), it can be found that the minimum is achieved if the optimal controls are specified as

$$
u_{k}^{o}=-\frac{1}{2 \rho_{k}} \frac{\partial V^{T}}{\partial \eta} B_{k} \eta, \quad k=1,2, \ldots ., m
$$

Under the specification of the optimal control $u_{k}^{o}$ in (51), the dynamic programming equation (50) becomes the following Hamilton-Jacobi equation (HJE)

$$
-\frac{\partial V}{\partial t}=\eta^{T} Q \eta+\frac{\partial V^{T}}{\partial \eta} A \eta-\sum_{k=1}^{m} \frac{1}{4 \rho_{k}} \frac{\partial V^{T}}{\partial \eta} B_{k} \eta \eta^{T} B_{k}^{T} \frac{\partial V}{\partial \eta}
$$

$V\left(\eta_{f}, t_{f}\right)=\eta_{f}^{T} F \eta_{f}$

Therefore Theorem 1 is proved.

\section{APPENDIX 2:}

Proof of Theorem 2:

By Bellman dynamic programming principle for nonlinear stochastic optimal control systems, we get

$$
\begin{aligned}
& -\frac{\partial V(\eta, t)}{\partial t}=\min _{u(t)} H\left(t, \eta, u, \frac{\partial V}{\partial \eta}, \frac{\partial^{2} V}{\partial \eta^{2}}\right) \\
& V\left(\eta_{f}, t_{f}\right)=\eta_{f}^{T} F \eta_{f}
\end{aligned}
$$

where $V(\eta, t)>0$ and the generalized Hamiltonian function $H$ is defined as

$$
\begin{aligned}
& H\left(t, \eta, u, \frac{\partial V}{\partial \eta}, \frac{\partial^{2} V}{\partial \eta^{2}}\right):=\eta^{T} Q \eta+\sum_{k=1}^{m} \rho_{k} u_{k}^{2}+ \\
& \frac{\partial V(\eta, t)^{T}}{\partial \eta}\left(A \eta+\sum_{k=1}^{m} u_{k} B_{k} \eta\right)+\frac{1}{2} \eta^{T} C^{T} \frac{\partial^{2} V(\eta, t)}{\partial \eta^{2}} C \eta \\
& =\left\{\eta^{T} Q \eta+\frac{\partial V^{T}}{\partial \eta} A \eta\right. \\
& +\sum_{k=1}^{m}\left(\rho_{k} u_{k}+\frac{1}{2} \eta^{T} B_{k}^{T} \frac{\partial V}{\partial \eta}\right)^{T} \frac{1}{\rho_{k}}\left(\rho_{k} u_{k}+\frac{1}{2} \eta^{T} B_{k}^{T} \frac{\partial V}{\partial \eta}\right)
\end{aligned}
$$

$$
\left.-\sum_{k=1}^{m} \frac{1}{4 \rho_{k}} \frac{\partial V^{T}}{\partial \eta} B_{k} \eta \eta^{T} B_{k}^{T} \frac{\partial V}{\partial \eta}+\frac{1}{2} \eta^{T} C^{T} \frac{\partial^{2} V(\eta, t)}{\partial \eta^{2}} C \eta\right\}
$$

Here the term $\frac{1}{2} \eta^{T} C^{T} \frac{\partial^{2} V(\eta, t)}{\partial \eta^{2}} C \eta$ appears due to the Itô differentiation in stochastic process [38-40]. Obviously, from (54), it follows that

$$
\min _{u(t)} H\left(t, \eta, u, \frac{\partial V}{\partial \eta}, \frac{\partial^{2} V}{\partial \eta^{2}}\right)=H\left(t, \eta, u_{k}^{o}, \frac{\partial V}{\partial \eta}, \frac{\partial^{2} V}{\partial \eta^{2}}\right)
$$

where

$$
u_{k}^{o}=-\frac{1}{2 \rho_{k}} \frac{\partial V^{T}}{\partial \eta} B_{k} \eta, \quad k=1,2, \ldots, m
$$

and the dynamic programming equation (53) becomes the following $\mathrm{HJE}$

$$
\begin{aligned}
& -\frac{\partial V}{\partial t}=\eta^{T} Q \eta+\frac{\partial V^{T}}{\partial \eta} A \eta- \\
& \sum_{k=1}^{m} \frac{1}{4 \rho_{k}} \frac{\partial V^{T}}{\partial \eta} B_{k} \eta \eta^{T} B_{k}^{T} \frac{\partial V}{\partial \eta}+\frac{1}{2} \eta^{T} C^{T} \frac{\partial^{2} V(\eta, t)}{\partial \eta^{2}} C \eta
\end{aligned}
$$

$V\left(\eta_{f}, t_{f}\right)=\eta_{f}^{T} F \eta_{f}$

Therefore Theorem 2 is proved.

\section{APPENDIX 3:}

Before proving Lemma 2, the following fact should be first proved.

Fact 1

$$
\left(\frac{\partial\left(\left(\otimes_{i} x\right)^{T} P\left(\otimes_{i} x\right)\right)}{\partial x}\right)^{T} C x=2\left(\left(\otimes_{i} x\right)^{T} Q\left(\otimes_{i} x\right)\right)
$$

where $P$ is symmetric and the components of $Q$ are of the following form [3]

$$
\mathrm{Q}_{k_{1}, \ldots \ldots, k_{i}}^{l_{1}, \ldots \ldots, l_{i}}=\sum_{m=1}^{i} \sum_{k_{m}=1}^{n} P_{k_{1}, \ldots, k_{m}, \ldots, k_{i}}^{l_{1}, \ldots, l_{i}} C_{k_{m} ; k_{m}}
$$

Proof of fact 1:

The right hand side of (58) can be expressed as follows :

$$
2\left(\left(\otimes_{i} x\right)^{T} Q\left(\otimes_{i} x\right)\right)=2 \sum_{\substack{l_{1}, w_{i} \\ k_{1} \cdots k_{i}}} x_{l_{1}} \cdots x_{l_{i}} Q_{k_{1} \cdots k_{i}}^{l_{1} \cdots l_{i}} x_{k_{1}} \cdots x_{k_{i}}
$$

Now we define the tensor $\delta_{h, k}=\left\{\begin{array}{lll}1 & \text { if } & h=k \\ 0 & \text { if } & h \neq k\end{array}\right.$.

Then the left hand side of (58) can be expressed as follows:

$$
\left(\frac{\partial\left(\left(\otimes_{i} x\right)^{T} P\left(\otimes_{i} x\right)\right)}{\partial x}\right)^{T} C x
$$




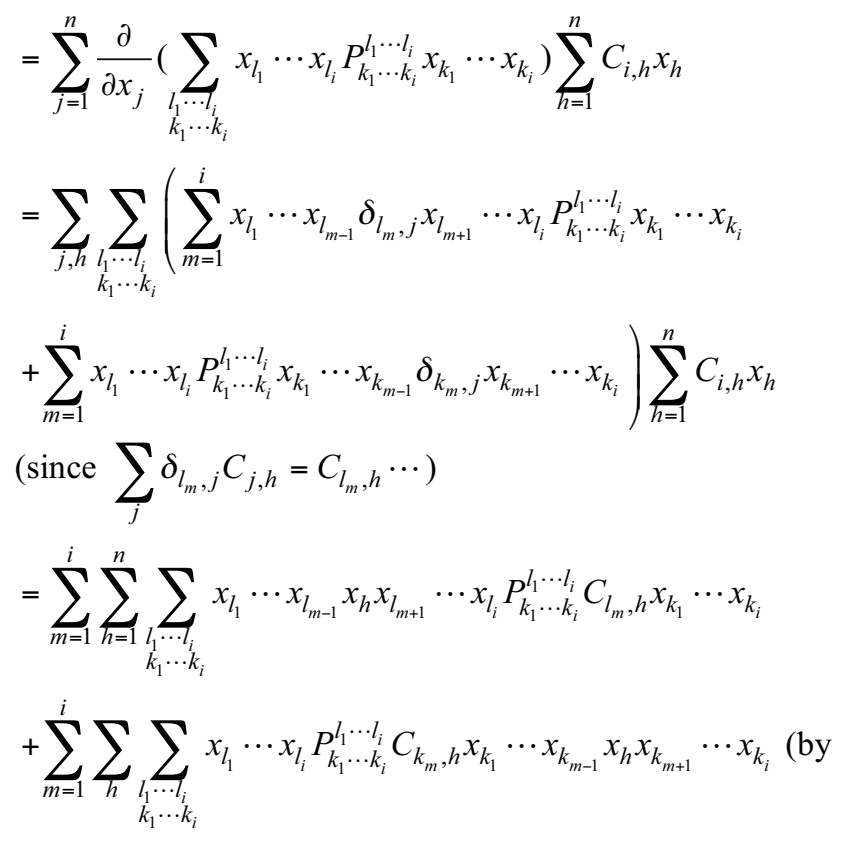

exchanging $\left.l_{m} \leftrightarrow h \cdots\right)$

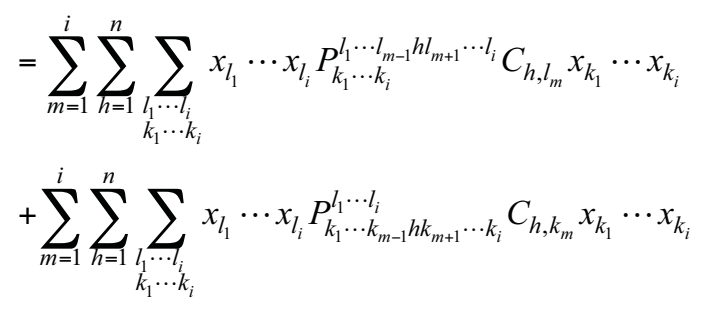

(by exchanging $l_{j} \leftrightarrow k_{j} \forall j$ in the first term and using $P$ symmetric)

$=2 \sum_{m=1}^{i} \sum_{h=1}^{n} \sum_{\substack{l_{1} \cdots l_{i} \\ k_{1} \cdots k_{i}}} x_{l_{1}} \cdots x_{l_{i}} P_{k_{1} \cdots k_{m-1} h k_{m+1} \cdots k_{i}}^{l_{1} \cdots l_{i}} C_{h, k_{m}} x_{k_{1}} \cdots x_{k_{i}}$

(by replacing $h$ with $k_{m^{\prime}}$ )

$$
\begin{aligned}
& =2 \sum_{m=1}^{i} \sum_{\substack{k_{m}=1 \\
l^{1} l_{1} \cdots l_{i} \\
k_{1} \cdots k_{i}}}^{n} x_{l_{1}} \cdots x_{l_{i}} P_{k_{1} \cdots k_{m-1} k_{m}{ }^{\prime} k_{m+1} \cdots k_{i}}^{l_{1} \cdots l_{i}} C_{k_{m}{ }^{\prime}, k_{m}} x_{k_{1}} \cdots x_{k_{i}} \\
& =2 \sum_{\substack{l_{1} \cdots l_{i} \\
k_{1} \cdots k_{i}}} x_{l_{1}} \cdots x_{l_{i}} \\
& \left(\sum_{m=1}^{i} \sum_{k_{m}=1}^{n} P_{k_{1} \cdots k_{m-1} k_{m}{ }^{\prime} k_{m+1} \cdots k_{i}}^{l_{1} \cdots l_{i}} C_{k_{m}{ }^{\prime}, k_{m}}\right) x_{k_{1}} \cdots x_{k_{i}}
\end{aligned}
$$

Comparing (60) and (61), we have proved (59).

Therefore the Fact 1 is proved.

Proof of (25):

After proving the equations (58) and (59), for the purpose of application, we need to factorize the matrix $Q$ to be $P \phi_{i}(C)$ as follows

$\frac{\partial\left(\left(\otimes_{i} x\right)^{T} P\left(\otimes_{i} x\right)\right)^{T}}{\partial x} C x=2\left(\left(\otimes_{i} x\right)^{T} Q\left(\otimes_{i} x\right)\right)$

$$
=2\left(\left(\otimes_{i} x\right)^{T} P \phi_{i}(C)\left(\otimes_{i} x\right)\right)
$$

So the components of the matrix $Q$ satisfy

$\mathrm{Q}_{k_{1}, \ldots \ldots, k_{i}}^{l_{1}, \ldots \ldots \ldots, l_{i}}=\sum_{q_{1}}^{n} \sum_{q_{2}}^{n} \ldots \sum_{q_{i}}^{n} P_{q_{1}, \ldots \ldots \ldots, q_{i}}^{l_{1}, \ldots \ldots, l_{i}}\left(\phi_{i}(C)\right)_{k_{1}, \ldots \ldots \ldots, k_{i}}^{q_{1}, \ldots \ldots \ldots, q_{i}}$

Comparing (63) with (59), we can find the element $\left(\phi_{i}(C)\right)_{k_{1}, \ldots, k_{i}}^{l_{1}, \ldots, l_{i}}$ satisfies

$\left(\phi_{i}(C)\right)_{k_{1}, \ldots \ldots, k_{i}}^{l_{1}, \ldots \ldots, l_{i}}=$

$\left\{\begin{array}{l}C_{k_{1}, k_{1}}+C_{k_{2}, k_{2}}+\ldots+C_{k_{i}, k_{i}} \text { if } l_{1}=k_{1}, l_{2}=k_{2}, \ldots ., l_{i}=k_{i} \\ C_{l_{j}, k_{j}} \text { if } l_{1}=k_{1}, \ldots l_{j-1}=k_{j-1}, l_{j+1}=k_{j+1}, \ldots, l_{i}=k_{i} \text { but } l_{j} \neq k_{j} \\ 0 \quad \text { others }\end{array}\right.$

Therefore Lemma 2 is proved.

For example, for a 2-dimensional state, we have

$$
\begin{aligned}
\phi_{1}(C) & =\left[\begin{array}{ll}
C_{1,1} & C_{1,2} \\
C_{2,1} & C_{2,2}
\end{array}\right], \\
\phi_{2}(C) & =\left[\begin{array}{cccc}
2 C_{1,1} & C_{1,2} & C_{1,2} & 0 \\
C_{2,1} & C_{1,1}+C_{2,2} & 0 & C_{1,2} \\
C_{2,1} & 0 & C_{2,2}+C_{1,1} & C_{1,2} \\
0 & C_{2,1} & C_{2,1} & 2 C_{2,2}
\end{array}\right] \\
\phi_{3}(C) & =\left[\begin{array}{ccccc}
3 C_{1,1} & C_{1,2} & C_{1,2} & 0 \\
C_{2,1} & 2 C_{1,1}+C_{2,2} & 0 & C_{1,2} & \\
C_{2,1} & 0 & 2 C_{1,1}+C_{2,2} & C_{1,2} & \\
0 & C_{2,1} & C_{2,1} & C_{1,1}+2 C_{2,2} & \\
C_{2,1} & 0 & 0 & 0 & \\
0 & C_{2,1} & 0 & 0 & \\
0 & 0 & C_{2,1} & 0 & \\
0 & 0 & 0 & C_{2,1} & \\
& C_{1,2} & 0 & 0 & 0 \\
& 0 & C_{1,2} & 0 & 0 \\
& 0 & 0 & C_{1,2} & 0 \\
& 0 & 0 & 0 & C_{1,2} \\
& 2 C_{1,1}+C_{2,2} & C_{1,2} & C_{1,2} & 0 \\
& C_{2,1} & C_{1,1}+2 C_{2,2} & 0 & C_{1,2} \\
& 0 & 0 & C_{1,1}+2 C_{2,2} & C_{1,2} \\
& & C_{2,1} & C_{2,1} & 3 C_{2,2}
\end{array}\right]
\end{aligned}
$$

\section{Appendix 4:}

Proof of Lemma 3:

$x^{T} C^{T} \frac{\partial^{2}\left(\left(\otimes_{i} x\right)^{T} P\left(\otimes_{i} x\right)\right)}{\partial x^{2}} C x=$

$x^{T} C^{T} \frac{\partial}{\partial x}\left(\left(\frac{\partial\left(\left(\otimes_{i} x\right)^{T} P\left(\otimes_{i} x\right)\right)}{\partial x}\right)^{T} C x\right)$

$-x^{T} C^{T}\left[\left(\frac{\partial\left(\left(\otimes_{i} x\right)^{T} P\left(\otimes_{i} x\right)\right)}{\partial x}\right)^{T} C\right]^{T} \quad$ (by $\quad$ using 


$$
-\left(\frac{\partial\left(\left(\otimes_{i} x\right)^{T} P\left(\otimes_{i} x\right)\right)}{\partial x}\right)^{T} C^{2} x
$$

(by using (24) and the fact that the symmetric part of $2 P \phi_{i}(C)$ is $\left.P \phi_{i}(C)+\phi_{i}(C)^{T} P\right)$

$=2\left(\otimes_{i} x\right)^{T}\left(\phi_{i}(C)^{T} P \phi_{i}(C)+P \phi_{i}(C)^{2}-P \phi_{i}\left(C^{2}\right)\right)\left(\otimes_{i} x\right)$ Therefore

Lemma 3 is proved.

For example, for a 2-dimensional state, we have

$\phi_{1}\left(C^{2}\right)=C^{2}=\left[\begin{array}{cc}C_{1,1}^{2}+C_{1,2} C_{2,1} & C_{1,1} C_{1,2}+C_{1,2} C_{2,2} \\ C_{2,1} C_{1,1}+C_{2,2} C_{2,1} & C_{2,1} C_{1,2}+C_{2,2}^{2}\end{array}\right]$,

$\phi_{2}\left(C^{2}\right)=$

$\left[\begin{array}{cc}2 C_{1,1}^{2}+2 C_{1,2} C_{2,1} & C_{1,1} C_{1,2}+C_{1,2} C_{2,2} \\ C_{2,1} C_{1,1}+C_{2,2} C_{2,1} & C_{1,1}^{2}+C_{1,2} C_{2,1}+C_{2,1} C_{1,2}+C_{2,2}^{2} \\ C_{2,1} C_{1,1}+C_{2,2} C_{2,1} & 0 \\ 0 & C_{2,1} C_{1,1}+C_{2,2} C_{2,1} \\ C_{1,1} C_{1,2}+C_{1,2} C_{2,2} & 0 \\ 0 & C_{1,1} C_{1,2}+C_{1,2} C_{2,2} \\ C_{2,1} C_{1,2}+C_{2,2}^{2}+C_{1,1}^{2}+C_{1,2} C_{2,1} & C_{1,1} C_{1,2}+C_{1,2} C_{2,2} \\ C_{2,1} C_{1,1}+C_{2,2} C_{2,1} & 2 C_{2,1} C_{1,2}+2 C_{2,2}^{2}\end{array}\right]$

\section{REFERENCES}

[1] T. J. Tarn, G. Huang and J. W. Clark, "Modeling of quantum mechanical control systems", Mathematical Modeling, vol.1, pp.109$121,1980$.

[2] G. Huang, T. J. Tarn and J. W. Clark, "On the controllability of quantum mechanical systems", Journal of Mathematical Physics, vol. 24, no.11, pp. 2608-2618, 1983.

[3] C. K. Ong, G. Huang, T. J. tarn, and J. W. Clark, "Invertibility of Quantum Mechanical Control Systems", Mathemaical System Theory, vol. 17, pp. 335-350, 1984.

[4] A. P. Peirce, M. A. Dahleh, and H. Rabitz, "Optimal control of quantum mechanical systems; existence, numerical approximation and application", Physical Review A, vol. 37, pp. 4950-4964, 1988.

[5] M. A. Dahleh, A. Peirce, and H. Rabitz, "Optimal control of uncertain quantum systems”, Physical Review A, vol. 42, pp. 1065-1079, 1990.

[6] S. Shi, A. Woody, and H. Rabitz,"Optimal control of selective vibrational excitation in harmonic linear chain molecules", Journal of Chemical Physics, vol. 88, pp. 6870-6883, 1988.

[7] S. Grivopoulos and B. Bamieh, "Iterative algorithms for optimal control of quantum systems", Proceeding of the 41 st IEEE conference on Decision and control, Las Vegas, Nevada, 2002, pp. 26872690 ,

[8] R. S. Judson and H. Rabitz, "Teaching laser to control molecules", Physical Review Letters, vol. 68, pp.1500-1503, 1992.

[9] R. J. Gordon and S. A. Rice, "Active control of the dynamics of atoms and molecules", Annual Review of Physical Chemistry, vol. 48, pp. 601-641, 1997.

[10] V. Ramakrishna, M. V. Salapaka, M. A. Dahleh, H. Rabitz, and A. P. Peirce, "Controllability of molecular systems", Physical Review $A$, vol. 51, pp. 960-966, 1995.

[11] D. D'Alessandro, "On the controllability of systems on compact Lie groups and quantum mechanical systems", Journal of Mathematical Physics, vol. 42, pp. 4488-4496, 2001.

[12] G. Turinici and H. Rabitz, "Quantum wave function controllability", Journal of Chemical Physics, vol. 267, pp. 1-9, 2001.
S. Shirmer, J. V. Leahy, and A. I. Solomon, "Degree of controllability for quantum systems and applications to atomic systems", Journal of Physics, vol. 35, pp. 4125-4144, 2002.

[14] Y. Yamamoto, N. Imoto, and S. Machida, "Amplitude squeezing in a semiconductor laser using quantum nondemolition measurement and negative feedback", Physical Review A, vol. 33, pp. 3243$3261,1986$.

[15] H. A. Haus and Y. Yamamoto, "Theory of feedback-generated squeezed states", Physical Review A, vol. 34, pp. 270-297, 1986.

[16] J. H. Shapiro et al., "Theory of light detection in the presence of feedback", Journal of the Optical Society of America B, vol. 4, pp. 1604-1620, 1987.

[17] M. Yanagisawa and H. Kimura, "Transfer function approach to quantum control part I: Dynamics of quantum feedback systems", IEEE Transactions on Automatic Control, vol. 48, no.12, pp. 2107$2120,2003$.

[18] M. Yanagisawa and H. Kimura, "Transfer function approach to quantum control part II: Control concept and applications", IEEE Transactions on Automatic Control, vol. 48, no.12, pp. 2121-2132, 2003.

[19] J. Dalibard, Y. Cartin, and K. Molmer, "Wave-function approach to dissipative processed in quantum optics", Physical Review Letters, vol. 68 , pp. 580-583, 1992.

[20] C. W. Gardiner, A. S. Parkins, and P. Zoller, "Wave function quantum stochastic differential equations and quantum jump simulation method", Physical Review A, vol. 46, pp. 4363-4381, 1992.

[21] H. J. Carmichael, "Quantum trajectory theory for cascaded open systems", Physical Review Letters, vol. 70, pp. 2273-2276, 1993.

[22] H. M. Wiseman, "Quantum theory of continuous feedback", Physical Review A, vol. 49, pp. 2133-2150, 1994.

[23] A. C. Doherty, S. Halil, K. Jacols, H. Mabuchi, and A. M. Tan, "Quantum feedback control and classical control theory", Physical Review A, vol. 62, pp. 012105, 2000.

[24] S. P. Banks, "Control Systems Engineering", Prentice-Hall, London, 1986.

[25] B. S. Chen and S. T. Chen, " $H_{\infty}$ control design in bilinear systems: a tensor formal series approach", Systems Control \& Letters, vol. 23, pp.15-26, 1994.

[26] D. D'Alessandro and M. Dahleh, "Optimal control of two-level quantum systems", IEEE Transactions on Automatic Control, vol. 46, no.6, pp.866-876, 2001.

[27] J. Gough, V.P. Belavkin, and O.G. Smolyanov. "Hamilton-JacobiBellman equations for quantum optimal feedback control", Journal of Optics B: Quantum and Semiclassical Optics, vol. 7, pp. S237S244, 2005.

[28] S. H. Tersigni, P. Gaspard and S. A. Rice, "On using shaped light pulses to control the selectivity of product formation in a chemical reaction: An application to a multiple level system", Journal of Chemical Physics, vol. 93, no.3, pp. 1670-1680, 1990.

[29] E. Merzbacher, "Quantum Mechanics", 3rd ed. Wiley, New York, 1998

[30] A.G. Butkovskii and Y.I. Samoilenko, "Control of QuantumMechanical Processes and Systems", Kluwer Academic, Dordrecht, 1990.

[31] A. Ferrante, M. Pavon, and G. Raccanelli, Eds. "Driving the propagator of a spin system: a feedback approach", Proceedings of the 41st Conference on Decision and Control, pp. 46-50, Las Vegas, NV, December 2002

[32] S. Grivopoulos and B. Bamieh, Eds. "Lyapunov-based control of quantum systems", Proceedings of the 42nd IEEE Conference on Decision and Control, Maui, Hawaii, 2003, pp. 434-438.

[33] M. Mirrahimi and P. Rouchon, Eds. "Trajectory tracking for quantum systems: a Lyapounov approach", Proceedings of the Sixteenth International Symposium on MTNS, 2004.

[34] F. Ticozzi, A. Ferrante and M. Pavon, Eds. "Stability and Robustness in Quantum Coherent Control", Proceedings of the 16th International Symposium on MTNS, 2004.

[35] R. V. Handel, J. K. Stockton and H. Mabuchi, "Feedback control of quantum state reduction", IEEE Transition Automatic Control, no. 50, pp. 768-780, 2005.

[36] S. P. Banks, "Mathematical Theories of Nonlinear Systems", Prentice-Hall, London, 1986

[37] L. E. Ballentine, "Quantum Mechanics", Prentice-Hall, New Jersey, 1990.

[38] G. Chen, G. Chen and S.H. Hsu, "Linear Stochastic Control Systems", CRC Press, London, 1995. 
[39] B. S. Chen and W. Zhang, "Stochastic $H_{2} / H_{\infty}$ control with state dependent noise", IEEE Transactions on Automatic Control, vol. 49, no.1, pp. 45-57, 2004.
[40] W. Zhang and B. S. Chen, " $H_{\infty}$ control for nonlinear stochastic systems", SIAM Journal on Control and Optimization, vol. 44, no. 6, pp. 1973-1991, 2006.

Received: April 10, 2008

(C) Chen et al.; Licensee Bentham Open.

This is an open access article licensed under the terms of the Creative Commons Attribution Non-Commercial License (http://creativecommons.org/licenses/by-nc/3.0/) which permits unrestricted, non-commercial use, distribution and reproduction in any medium, provided the work is properly cited. 